\title{
Endometrial extracellular matrix rigidity and IFN $\tau$ ensure the establishment of early pregnancy through activation of YAP
}

\author{
Tao Zhang ${ }^{1}$ | Shuai Guo ${ }^{1}$ | Han Zhou ${ }^{1}$ | Zhimin $\mathbf{W u}^{1}$ | Junfeng Liü ${ }^{2}$ | \\ Changwei Qiu ${ }^{1}$ (i) | Ganzhen Deng ${ }^{1}$ (I)
}

${ }^{1}$ Department of Clinical Veterinary Medicine, College of Veterinary Medicine, Huazhong Agricultural University, Wuhan, China

${ }^{2}$ College of Animal Science, Tarim University, Alar, China

\section{Correspondence}

Ganzhen Deng, College of Veterinary Medicine, Huazhong Agricultural University, Wuhan 430070, China.

Email:925651088@qq.com

Funding informationThis study was supported by the National Natural Science Foundation of China (31772816).

\begin{abstract}
Background: In mammals, early pregnancy is a critical vulnerable period during which complications may arise, including pregnancy failure. Establishment of a maternal endometrial acceptance phenotype is a prerequisite for semiheterogeneous embryo implantation, comprising the rate-limiting step of early pregnancy.

Methods: Confocal fluorescence, immunohistochemistry and western blot for nuclear and cytoplasmic protein were used to examine the activation of yes-associated protein (YAP) in uterine tissue and primary endometrial cells. The target binding between miR16a and YAP was verified by dual-luciferase reporter gene assay. The mouse pregnancy model and pseudopregnancy model were used to investigate the role of YAP in the maternal uterus during early pregnancy in vivo.

Results: We showed that YAP translocates into the nucleus in the endometrium of cattle and mice during early pregnancy. Mechanistically, YAP acts as a mediator of ECM rigidity and cell density, which requires the actomyosin cytoskeleton and is partially dependent on the Hippo pathway. Furthermore, we found that the soluble factor IFN $\tau$, which is a ruminant pregnancy recognition factor, also induced activation of YAP by reducing the expression of miR-16a.

Conclusions: This study revealed that activation of YAP is necessary for early pregnancy in bovines because it induced cell proliferation and established an immunosuppressive local environment that allowed conceptus implantation into the uterine epithelium.
\end{abstract}

\section{1 | INTRODUCTION}

Although advances in reproductive science over the past few decades have provided improved our knowledge of clinical conditions during pregnancy, the incidence of infertility and pregnancy complications has greatly increased worldwide. ${ }^{1-3}$ Many scientists have focused on elucidating the physiological mechanisms that occur during pregnancy, investigating the molecular expression network, and understanding space-and time-specific expression patterns to address reproductive diseases. However, our knowledge of embryos and the maternal uterus during early pregnancy in mammals remains limited, due to ethical considerations, technical limitations and high research costs. ${ }^{4,5}$ Integrating the information gained from humans and other mammals during pregnancy is important for overcoming these difficulties. 
In mammalian pregnancies, embryo implantation is a rate-limiting step and critical vulnerable period during which complications arise and pregnancy failure occurs. ${ }^{6}$ A prerequisite for successful implantation is an intimate maternal-foetal dialogue between the receptive uterine lining of the mother and the specialized trophectodermal cells of the embryo and this dialogue can be mediated by biochemical signals such as nucleic acids and hormones, as well as physical signals. ${ }^{6-8}$ It is widely accepted that soluble biochemical signals secreted by the conceptus, such as human chorionic gonadotropin (HCG) and IFN $\tau$ secreted by humans and ruminants, respectively, are required for pregnancy recognition. ${ }^{9-11}$ Well-timed modification of mechanical parameters in the uterus during pregnancy is key to a successful pregnancy, including establishing an elastic fibre network, cell polarity, and matrix stiffness. ${ }^{12-14}$ Few studies have been performed on the molecular mechanistic changes caused by uterine mechanical behaviour during pregnancy. The response of the maternal uterus to the embryo is similar during early pregnancy in mammals, including decidualization and local immunosuppression. Therefore, further studies are necessary to uncover how the maternal uterus responds to biochemical and mechanical factors during early pregnancy, especially because this molecular mechanism may be evolutionarily conserved.

The Hippo pathway, which tightly regulates organ size and regeneration by restricting cell proliferation and stem cell self-renewal, is an evolutionarily conserved protein kinase cascade in mammals. ${ }^{15-17}$ Recent findings indicate that the Hippo pathway is activated not only through soluble signals (eg, hormone and protein signals) $)^{17,18}$ but also through physical and mechanical cues, such as cell-cell junctions and the extracellular matrix (ECM). ${ }^{18,19}$ The chemical and physical properties of hormonal and mechanical forces are integral to morphogenetic processes during embryonic development and endometrial decidualization, driving specific cell differentiation programs. ${ }^{20}$ To date, multiple studies on the Hippo pathway have focused on the differentiation of embryonic stem cells. ${ }^{21,22}$ In contrast, little research has focused on whether and how the Hippo pathway controls the maternal uterus during early pregnancy. In this study, we report the identification of YAP in the maternal uterus as sensor and mediator of embryo signals that maintain early pregnancy.

\section{2 | MATERIALS AND METHODS}

\section{1 | Reagents and plasmids}

Bovine IFN $\tau$ and IL-6 were purchased from Cloud Clone Corp (Wuhan, China). Verteporfin was purchased from Macklin Biochemical (Shanghai, China, V873898). Latrunculin A (Lat. A) was purchased from Santa Cruz Biotechnology (sc-202691). Phalloidin was purchased from Sigma (P5282). miR-16a mimics/inhibitors/NC and siYAP/LATS1/2/NC were generated by GenePharma (Shanghai, China). Their sequences are provided in Table S1. The reporter plasmids pGLO-YAP-3'UTR, pGLO-YAP-3'UTR-MUT, pGLO-NC (Promega) and the overexpression plasmid pcDNA3.1(+) -YAP were ordered from GeneCreate (Wuhan, China, Project Nos. 1915 311, 1915312 and 1916340 ).

\section{2 | Bovines endometrial sample collection}

Healthy cattle uteri were collected from a local commercial abattoir that had slaughtered cattle within the previous $30 \mathrm{~min}$. The slaughterhouse is recognized by the Animal Welfare Organization and complies with the animal experiment rules of the Huazhong Agricultural University Animal Care and Use Committee. Non-pregnant uteri ( $n=10$, oestrous) and uteri during early pregnancy $(n=25,20-60 d)$ were collected three times after washing with sterile PBS and then immediately transported to the laboratory on ice or fixed in $4 \%$ formaldehyde solution. Normal, healthy uterine horns were soaked in PBS containing $10 \%$ antibiotic/antimycotic solution after collection.

\section{3 | Bioinformatics analysis}

Gene expression data and supplemental RNA-seq data for endometria during pregnancy were downloaded from the GEO database (GSE107891). We obtained DEGs from the GEO database using the $R$ package 'edgeR'. Here, genes with an adjusted $P$ value $<.01$ and a fold change $(F C)>1.5$ or $<-1.5$ were considered DEGs. In our study, the R package 'clusterprofiler' was used to provide functional classification of downregulated DEGs. We listed the top 10 terms in every category, and $P<.05$ was set as the cutoff point.

\subsection{Primary culture}

Bovine endometrial epithelial cells (bEECs) and endometrial stromal cells (bESCs) were isolated and cultured as previously described. ${ }^{23}$ In brief, uteri with no gross evidence of genital disease or microbial infection were soaked in PBS on ice for 1 hour until further processing in the laboratory. The tissue was digested in $20-25 \mathrm{ml}$ of DPBS (HyClone) sterile digestive solution, which contained $1 \%$ protease (Sigma, P5147) and 1\% collagenase II (Sigma-Aldrich). Following a 2 hour incubation in a shaking water bath at $37^{\circ} \mathrm{C}$, the cell suspension was filtered through a $40-\mu \mathrm{m}$ mesh (Fisher Scientific, Loughborough, UK) to remove undigested material. The suspension was centrifuged at $1000 \mathrm{r} / \mathrm{min}$ for 5 minutes, and after three additional washes in washing medium, the cells were resuspended in DMEM/F-12 culture medium containing 15\% FBS (Gibco), $50 \mathrm{IU} / \mathrm{ml}$ penicillin, $50 \mathrm{IU} / \mathrm{ml}$ of streptomycin, and $2.5 \mu \mathrm{g} / \mathrm{ml}$ of amphotericin $\mathrm{B}$ (all from HyClone). The cells were cultured in 6 or 12-well plates (Corning) or $25-$ or $75-\mathrm{cm}^{2}$ flasks at $37^{\circ} \mathrm{C}$. The endometrial epithelial and stromal cell populations were isolated by differential adhesion to cell culture flasks. Immunofluorescence staining for the endometrial epithelial cell marker CK18 and the stromal cell vimentin marker was performed to detect cell purity. Primary cells were used in the next experiment after four passages. 


\subsection{Cell culture, transfections and treatments}

Human HEK293 cells were purchased from American Tissue Culture Collection (ATCC). The cells were cultured under $5 \% \mathrm{CO}_{2}$ in ambient $\mathrm{O}_{2}$ at $37^{\circ} \mathrm{C}$ in RPMI 1640 medium (Invitrogen) containing 10\% foetal bovine serum (FBS, PAN, Germany).

2D culture on hydrogels with high (40 kPa) or low (1 kPa) stiffness was performed as described elsewhere. ${ }^{24}$ In brief, $10 \mu \mathrm{g} / \mathrm{ml}$ bovine fibrinogen (Solarbio) was used to coat hydrogels activated with SulfoSANPAH (Thermo Fisher Scientific) according to the needs of the cells.

bEECs at $60 \%$ confluence were exposed to $0.5 \mu \mathrm{M}$ Lat. A, 10, 50, or $100 \mathrm{ng} / \mathrm{ml} \mathrm{IFN} \tau$ or an equal volume of PBS for 24 hour and then cells were collected and stored at $-20^{\circ} \mathrm{C}$ for the next experiment. Transient transfection of HEK293 and primary cells was carried out using Lipofectamine 2000 (Invitrogen) as recommended by the manufacturer. Briefly, Lipofectamine 2000 and the plasmids, siRNA or miR-16a mimics/inhibitor/NC were separately dissolved in $500 \mu \mathrm{l}$ of Opti-MEM I medium (Gibco), and then these solutions were mixed and incubated for $20 \mathrm{~min}$ at room temperature to form complexes. The prepared mixture was added to exponentially growing cells $\left(4 \times 10^{5}\right)$ seeded in a 6-well plate for 12 hours. All transfections were performed in triplicate, and the cells were cultured in OPTI-MEM medium for 24 hour for RNA studies, or 48 hour for protein studies.

\subsection{Mice and treatment}

Female (aged 6-8 weeks) and male (aged 8-10 weeks) Kunming (KM) mice were purchased from the Laboratory Animal Service Centre of Huazhong Agricultural University. All mouse experiments were conducted in accordance with the university guidelines on animal experimentation (HZAUMO-2015-12), and approval by the Animal Ethics Committee of Huazhong Agricultural University was obtained for all related procedures. The mice were maintained at room temperature with a 12-h dark-light cycles and free access to food and water.

For the normal early pregnancy model, sexually mature female KM mice aged 6-7 weeks were randomly paired with a KM male mouse aged 8-10 weeks at 7:00 PM, and female mice that did not mate during the oestrous period were used as the non-pregnant group. The day a vaginal plug was observed the next morning after mating, at 8:00 AM was designated day 0.5 of pregnancy (ED 0.5). Uterine tissue was collected from normal pregnant mice at ED 0.5, ED 2.5, ED 4.5 and ED 8.5. For the pregnancy interference model, on ED 1.5, mice were intraperitoneally injected with verteporfin (VP, $10 \mu \mathrm{mol} / \mathrm{kg}$ ) and an equal amount of PBS (control) every two days or on ED 2.5, mice were intrauterine injection with $50 \mu \mathrm{L}$ siYAP/siNC (30 pmol) combined with Lipofectamine. Mice were euthanized on ED 7.5, and the uterus was imaged and weighed immediately. For intrauterine injection of the IFN- $\tau$ model described previously, female mice were randomly divided into two groups: (1) control and (2) IFN- $\tau$, in which IFN- $\tau$ (10 mg/kg, $20 \mu l)$ was intrauterine injected into mice every two days. The control group was injected with an equal volume of PBS. The mouse uterus was collected on the seventh day for further analysis. Then the uterus was flash frozen in liquid nitrogen and stored at $-80^{\circ} \mathrm{C}$ or fixed in $4 \%$ formaldehyde for subsequent analysis. All experimental and control groups contained 5 to 10 mice each.

The oil-induced decidualization mouse model was adapted as previously reported. ${ }^{3}$ Pseudopregnancy was induced in sexually mature females that were each randomly paired with vasectomized males, and the day a vaginal plug was observed was designated as day 0.5 of pseudopregnancy. Intrauterine injection of $25 \mu \mathrm{l}$ oil into one uterine horn was performed on day 3.5 of pseudopregnancy, and the contralateral uninjected horn served as a control $(n=6)$. The uterus was photographed and weighed on day 7.5.

\subsection{Dual-luciferase assays}

293T cells were plated onto 6-well plates at a density of $4 \times 10^{5}$ cells/ $\mathrm{ml}$ per well and cotransfected with pGLO-YAP or pGLO-YAP MUT and miR-16a mimics using Lipofectamine 2000. The luciferase activity was assessed using the Dual-Glo luciferase assay kit (Promega), according to the manufacturer's protocol. The cells were then lysed to measure luciferase activity according to the manufacturer's instructions.

\section{8 | Cell proliferation assay}

Cell proliferation was evaluated using CCK-8 (Dojindo Laboratories) assays according to the manufacturer's instructions. In brief, cells were seeded in 96-well plates at a density of $3 \times 10^{4}$ cells $/ \mathrm{ml}$, and five repetitions were performed for each group. After treatment, the cells were continuously cultured with $10 \mu \mathrm{L}$ of CCK-8 in each well at $37^{\circ} \mathrm{C}$ for 2 hours. Cell proliferation was measured through absorbance (optical density) at $450 \mathrm{~nm}$ with a microplate reader (Bio-Rad Instruments).

\section{9 | Gene expression analysis by RT-qPCR}

Total RNA was isolated using TRIzoI ${ }^{\circledR}$ reagent (Invitrogen) and cDNA was synthesized using the HiScript ${ }^{\circ}$ II Q Select RT SuperMix for qPCR kit (Vazyme Biotech Co., Ltd). Quantitative RT-qPCR was performed in duplicate using FastStart Universal SYBR Green Master Mix (Roche Applied Science) using the StepOne real-time PCR System (Life Technologies Corp.). The expression levels of miRNA were assessed using a Hairpin-itTM microRNA qPCR Quantitation Kit (GenePharma) according to the standard protocol. Expression levels are given relative to GAPDH or U6. Sequences of primers are provided in Tables S2 and S3.

\subsection{0 | Total, nuclear and cytoplasmic protein extraction}

Total protein from cells or tissue was extracted according to the protein extraction kit (Vazyme). Nuclear and 
(A)

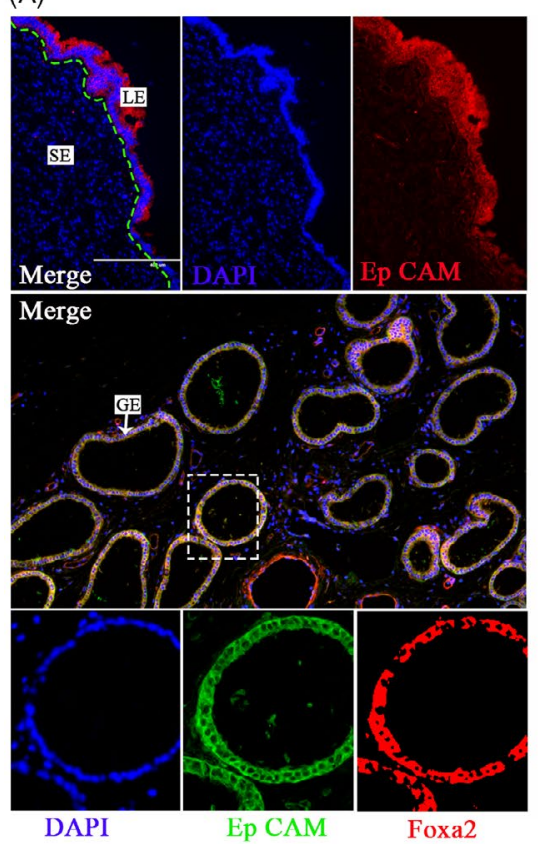

(B)

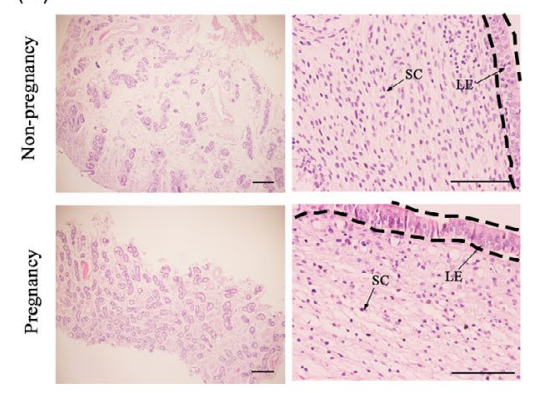

(C)
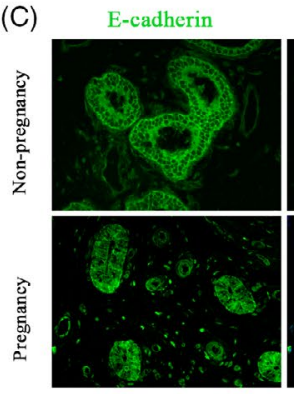

(D)

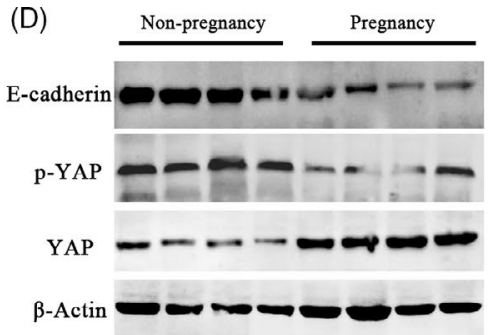

(E)

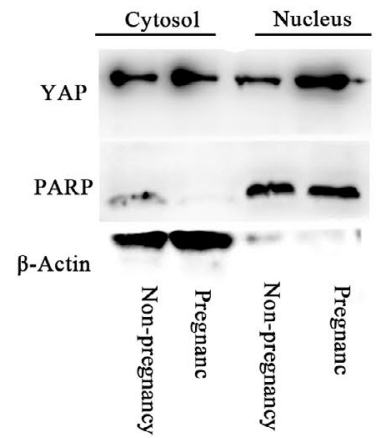

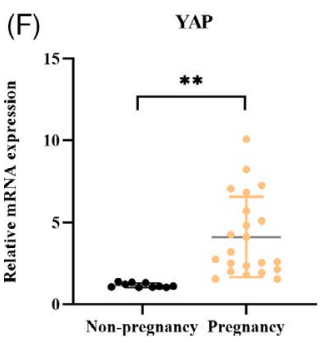

(G)
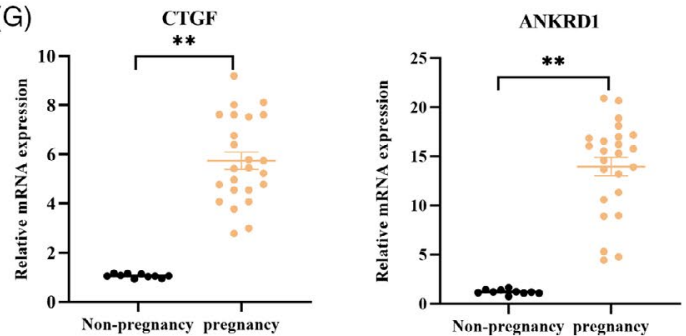

(I)

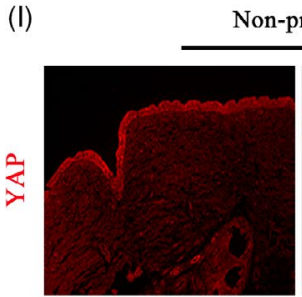

Non-pregnancy
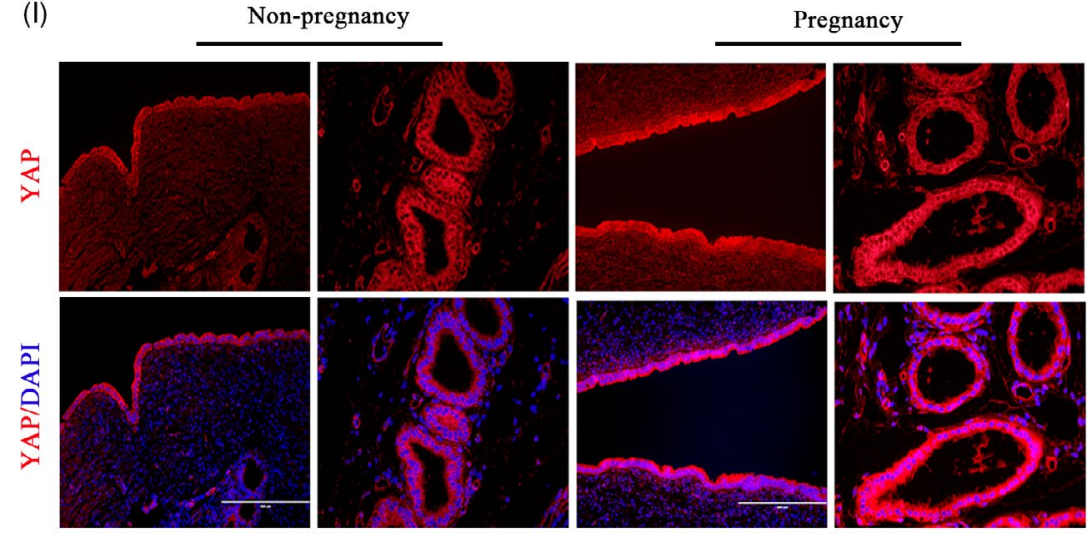

(H)
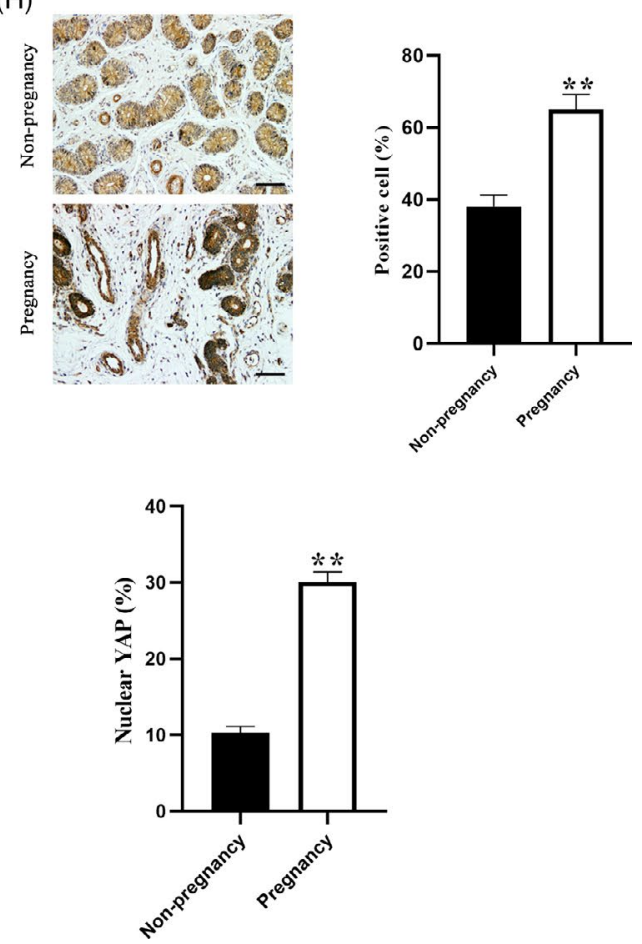

FIGURE 1 YAP is activated in the endometrium of pregnant bovines. A, Representative bovine uterine epithelia labelled with EpCAM antibody (epithelial marker), FOXA2 antibody (glandular-specific marker), and DAPI (nuclei) in a uterine tissue section. Higher magnification of the glandular tissue indicated in the dotted square in the lower merge panel is shown in the lower panel. Scale bars: $200 \mu \mathrm{m}$ (up), $400 \mu \mathrm{m}$ (medium) and $20 \mu \mathrm{m}$ (lower). B, Representative histopathological images from uterine tissue sections in pregnant and non-pregnant cows. Scale bars: 400 (Left) and $50 \mu \mathrm{m}$ (Right). C, E-cadherin visualized by immunostaining (green); nuclei were counterstained with DAPI (blue), $\mathrm{n}=2$. Scale bars, $50 \mu \mathrm{m}$. D, Protein expression of E-cadherin, YAP and $\mathrm{p}$-YAP in bovine uterine tissue by western blot, $\mathrm{n}=3$. $\mathrm{E}, \mathrm{Western}$ blotting for YAP in nuclear and cytoplasmic protein fractions from bovine endometrium, $n=3$. F, G, mRNA levels of YAP ( $f$ ), CTGF and ANKRD1 $(\mathrm{g})$ in bovine endometrium were detected by RT-qPCR, $n=3$. H, Representative immunohistochemical images (left, $n=2$ ) and quantification of YAP positive cells (right, $n=3$ ). Scale bars $200 \mu \mathrm{m}$. I, Representative immunofluorescence $(n=2)$ and quantifications of nuclear and cytoplasmic subcellular localization of YAP (right, $n=15$ ) in bovine endometrial epithelial cells. Scale bars $100 \mu \mathrm{m}$. Experiments were repeated $n$ times with two biological replicates. Data are shown as the mean \pm SEM. $P$ values were determined by an unpaired twosided t test. ${ }^{* *} P<.001$. See also Figure $\mathrm{S} 1$ 
(A)
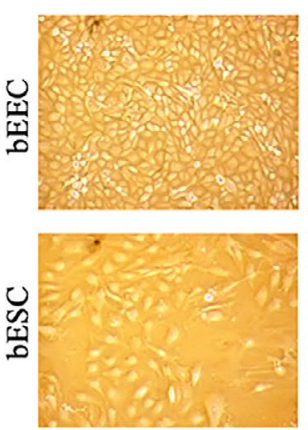

(D)
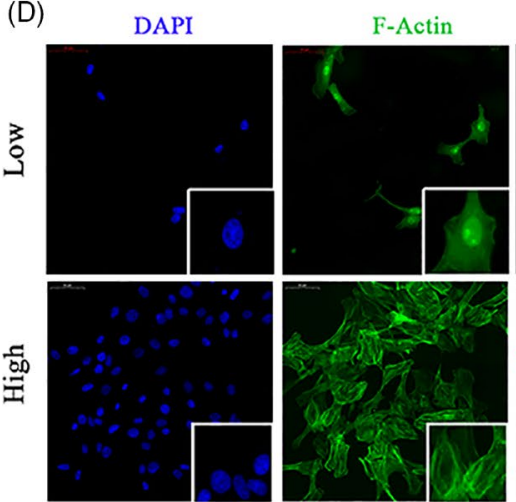

(F)
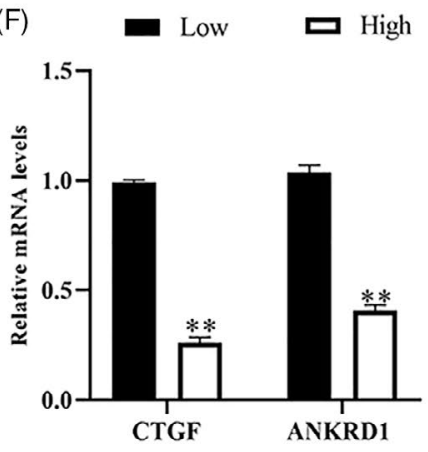

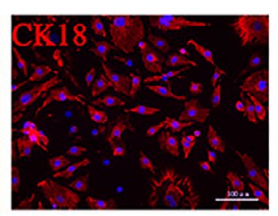

(B)

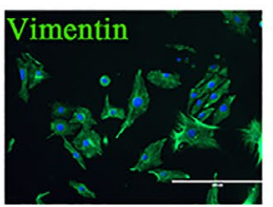

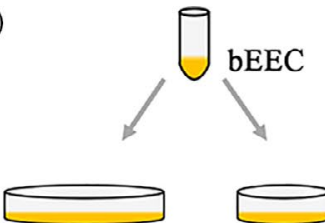

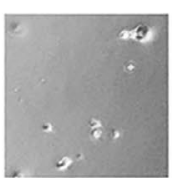

Low

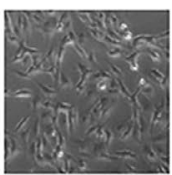

High

WILEY

(C)

density

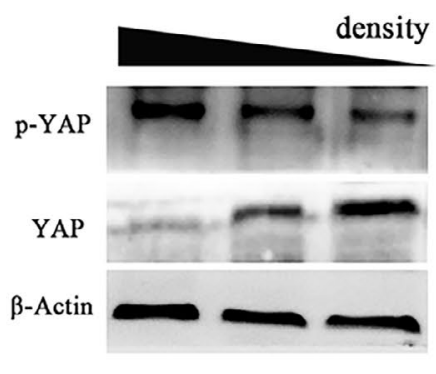

(E)
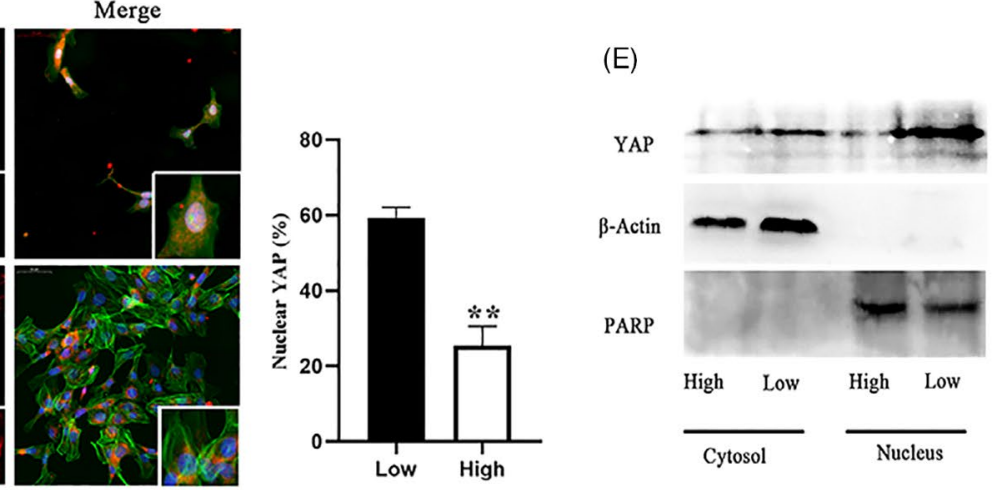

(G)
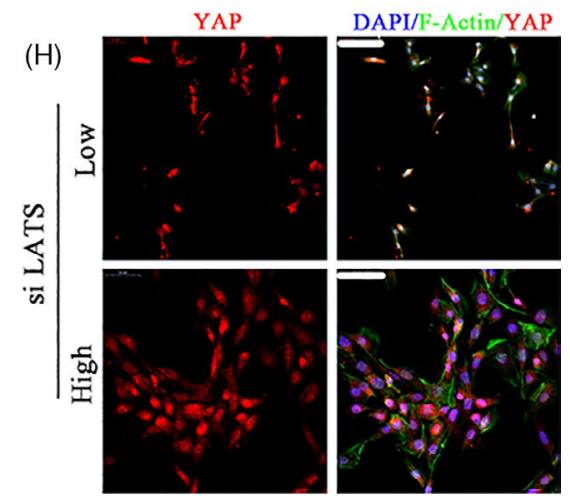

FIGURE 2 YAP is regulated by cell density in a manner dependent on the Hippo pathway. A, Images of primary cultured bovine endometrial cells (left) and immunofluorescence images corresponding to cell type markers (right). bEECs, bovine endometrial epithelial cells, CK18; bESCs, bovine endometrial stromal cells, vimentin. Scale bars, $100 \mu \mathrm{m}$ and $50 \mu \mathrm{m}, \mathrm{n}=3$. B, Schematic representation of different bEEC density culture methods and images of actual effects. The same number of cells were seeded in culture plates of different sizes. C, Protein expression of YAP and p-YAP in bEECs at different cell densities, $n=3$. D, Immunofluorescence images (left, $n=2$ ) and quantification of the nuclear and cytoplasmic subcellular localization of YAP (right, $n=15$ ) in bEECs, when grown at low/high densities. Scale bars, $50 \mu \mathrm{m}$. Enlarged $=10 \mu \mathrm{m}$. E, YAP expression in the cytosol and/or nucleus of bEECs grown at low/high density, $n=3 . \mathrm{F}$, mRNA levels of CTGF and ANKRD1 in bEECs cultured at low/high density were detected by RT-qPCR, $n=3$. G, Protein expression of YAP in bEECs treated with siNC or siLATS at low/high density, $\mathrm{n}=3$. H, Representative immunofluorescence of YAP in bEECs treated with siLATS at low/high densities, $\mathrm{n}=2$. Scale bars, $50 \mu \mathrm{m}$. DAPI, blue, nuclei; F-actin, green, cell boundaries; YAP, red. All experiments were repeated independently three times with similar results, $n=3$. Experiments were repeated $n$ times with two biological replicates. Data are shown as the mean \pm SEM. $P$ values were determined by an unpaired two-sided $t$ test. ${ }^{* *} P<.001$. See also Figure S2A-C

cytoplasmic proteins were extracted according to the Nuclear and Cytoplasmic Protein Extraction Kit (Sangon Biotech). The protein concentration was determined using a BCA protein assay kit (Vabzyme). $\beta$-actin and PARP were used to assess the purity of the cytoplasmic fraction and the nuclear fraction respectively.

\subsection{1 | Western blot analysis}

The protein was separated by SDS-PAGE (5\%-12\%), transferred onto $0.45 \mu \mathrm{m}$ PVDF membranes (Solarbio) and blocked in 10\% non-fat milk in TBST for 2 hour at room temperature. The blots were successively incubated with primary antibodies overnight at $4^{\circ} \mathrm{C}$. Following three washes 
with TBST, the membranes were then incubated with secondary antibodies at room temperature for 2 hour. After three washes, the membranes were subjected to chemiluminescence using Clarity Western ECL Substrate (Affinity). Anti- $\beta$-actin was used as control. Protein expression was detected using an enhanced chemiluminescence detection system (ImageQuant LAS 4000 mini, USA). Antibody information is provided in Table S4.

\subsection{2 | Immunofluorescence}

Cells grown on glass coverslips were fixed with $4 \%$ paraformaldehyde for 15 min at room temperature, washed three times with PBS and then permeabilized with $0.05 \%$ Triton X-100 for 10 min at room temperature. Following three 5 min washes in PBS, the coverslips were

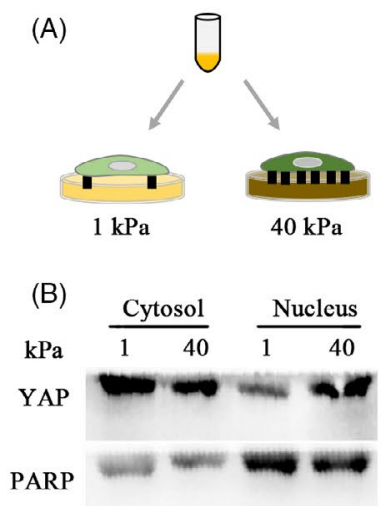

$\beta$-Actin

(D)

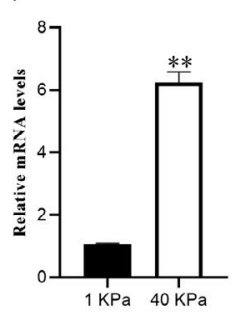

(C)
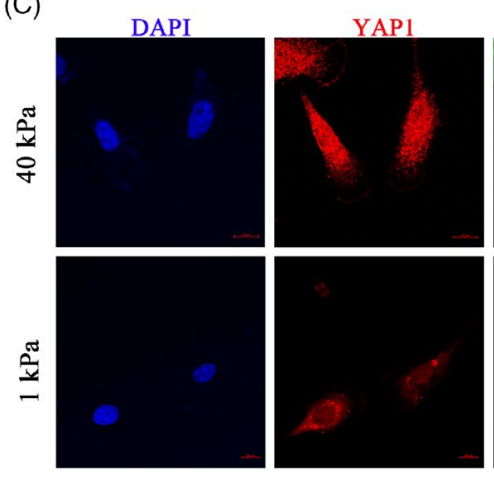
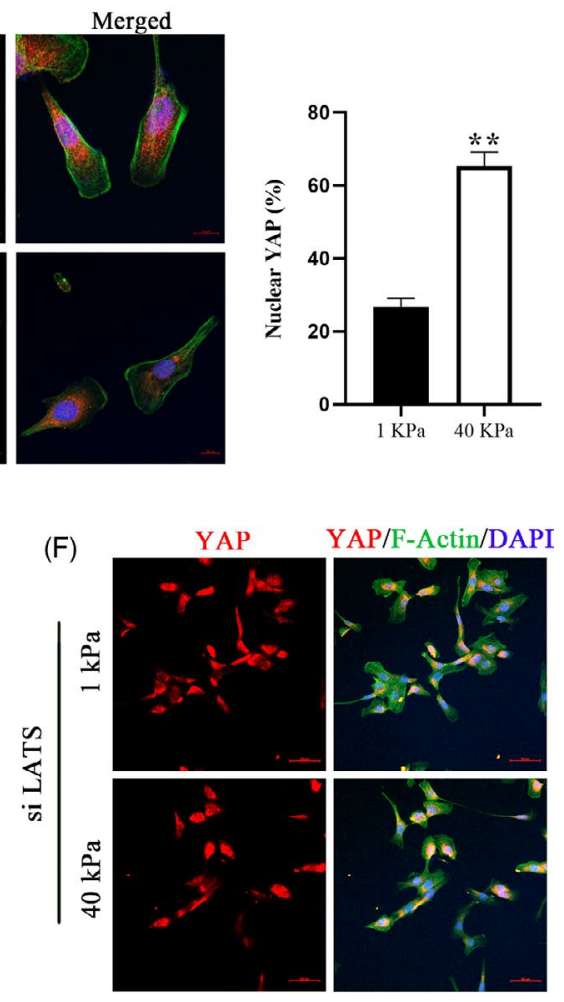

(G)

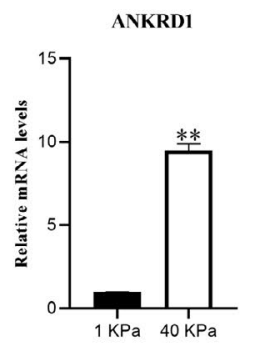

(E)
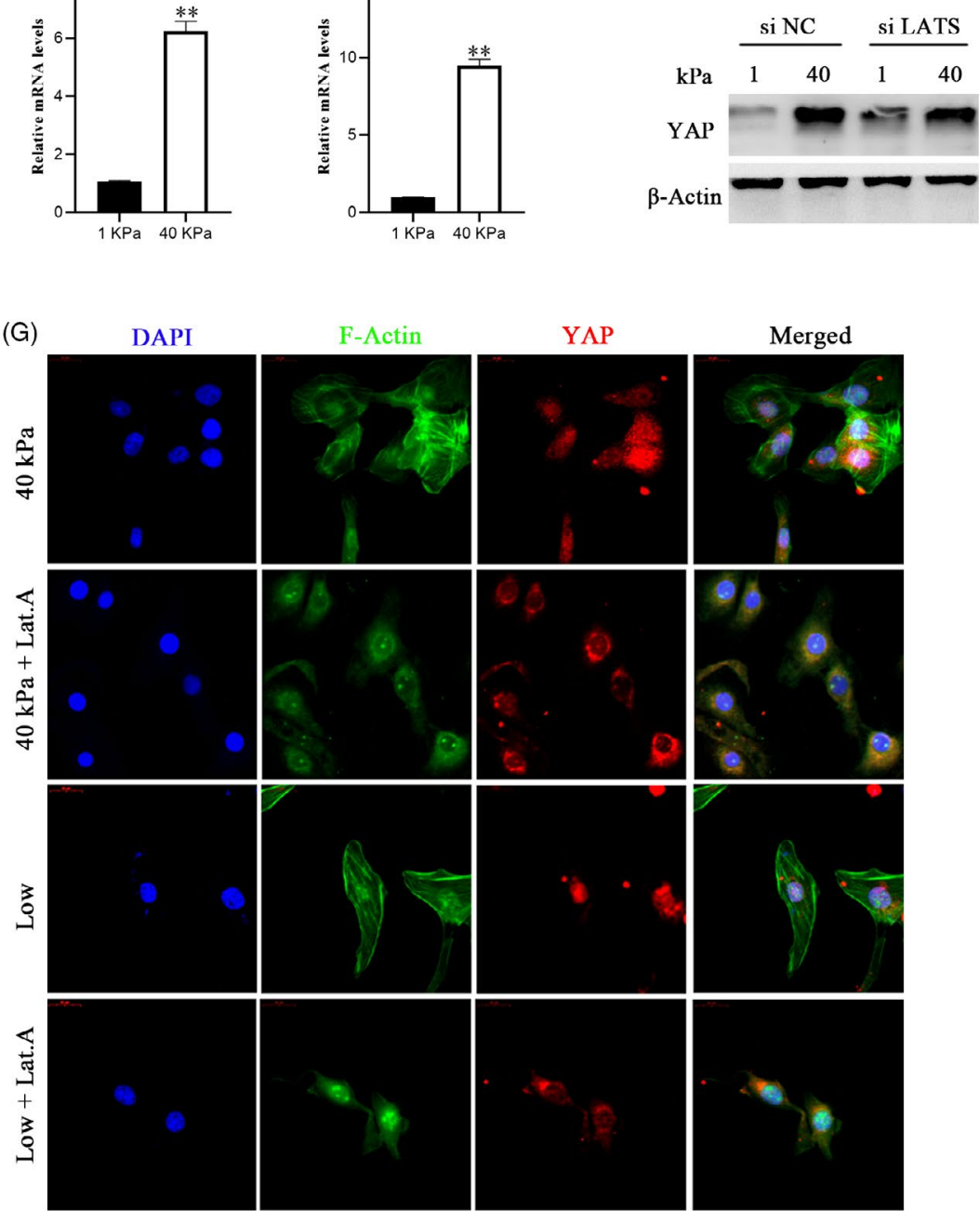
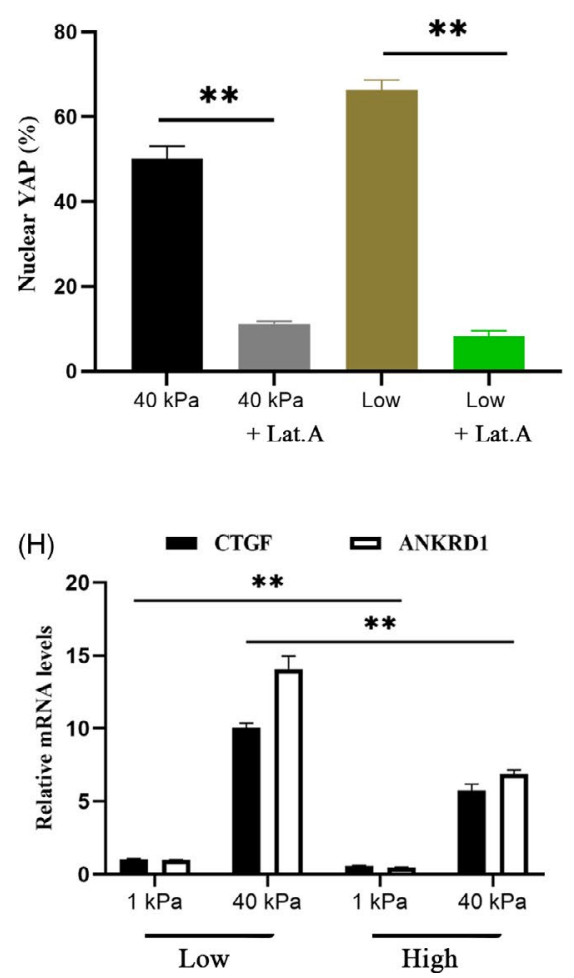
FIGURE 3 YAP is regulated by ECM stiffness independent of the Hippo pathway and requires tension of the actin cytoskeleton. a. Schematic representation of bEECs plated on hydrogels with different rigidities (40/1 kPa). B, Western blotting for YAP in nuclear and cytoplasmic protein fractions from $\mathrm{bEECs}$ plated on $40 \mathrm{kPa}$ and $1 \mathrm{kPa}$ fibronectin-coated hydrogels for 48 hour, $\mathrm{n}=3$. C, Confocal immunofluorescence images (left, $n=2$ ) and quantifications of nuclear and cytoplasmic subcellular localization (right, $n=15$ ) of YAP in bEECs plated on hydrogels with different rigidities. Scale bars, $10 \mu \mathrm{m}$. D, RT- qPCR analysis of mRNA levels of CTGF and ANKRD1 in bEECs plated on hydrogels with different rigidities, $n=3$. E, Protein expression of YAP in bEECs transfected with siNC or siLATS at 40/1 kPa hydrogels, $n=3$. $F$, Representative immunofluorescence of YAP in bEECs transfected with si LATS and cultured on hydrogels, $\mathrm{n}=2$. Scale bars, $50 \mu \mathrm{m}$. G, Confocal immunofluorescence images (left, $n=2$ ) and quantification of nuclear and cytoplasmic subcellular localization (right, $n=15$ ) of YAP in bEECs plated at $40 \mathrm{kPa}$ or low density. Cells were also treated with the F-actin inhibitor latrunculin A (Lat.A, $0.5 \mu$ M) or PBS (control) for 24 hour. Scale bars, $20 \mu \mathrm{m}$. h. RT-qPCR of bEECs grown under low or high conditions on the indicated hydrogels, $\mathrm{n}=3$. Experiments were repeated $n$ times with two biological replicates. Data are shown as the mean \pm SEM. $P$ values were determined by an unpaired two-sided $t$ test (c, $d, g$ ) and twoway ANOVA (h). ${ }^{* *} P<.001$. DAPI, blue, nuclei; F-actin, green, cell boundaries; YAP, red. See also Figure S2d-h

blocked with 5\% BSA for 30 min and then incubated overnight with antibodies against YAP, E-cadherin and $\mathrm{Ki} 67$ at $4^{\circ} \mathrm{C}$. Following three 5 min washes in PBST, the coverslips were incubated with secondary antibodies for 1 hour at room temperature in the dark. Following three 5 min washes with PBST, the coverslips were stained with DAPI. Images were obtained using an Imager Nikon Eclipse C1 (Japan), a Nikon DS-U3 (Carl Zeiss) or an optical microscope (Olympus).

\subsection{3 | Histology and immunohistochemical analysis}

Maternal uteruses were extracted from pregnant female cows or mice. The uterus was fixed in $4 \%$ formaldehyde solution and the tissue was embedded in paraffin. Sections $(4 \mathrm{~mm})$ were cut and stained with haematoxylin and eosin. Immunohistochemistry was performed on 4 micron-thick, FFPE sections using an anti-YAP antibody. FFPE sections were deparaffinized using xylene and rehydrated in graded ethanol. Sections were treated in $1 \mathrm{mM}$ EDTA, $\mathrm{pH}$ 8.0 by boiling at $125^{\circ} \mathrm{C}$ for 30 seconds and $90^{\circ} \mathrm{C}$ for 10 seconds inside a cocker within a microwave for antigen retrieval. All sections were incubated with endogenous peroxidase with $1 \% \mathrm{H}_{2} \mathrm{O}_{2}$ for 10 minutes and protein blocking reagents for 5 minutes each. The sections were then incubated with YAP antibody diluted in TBST diluent for 1 hour at room temperature. Following primary antibody incubation, the sections were incubated with monoclonal mouse anti-rabbit immunoglobulins for 1 hour at room temperature. Afterwards, the sections were washed with PBS and then counterstained with haematoxylin. Images were obtained using an Imager Nikon Eclipse C1, a Nikon DS-U3 (Carl Zeiss) or an optical microscope (Olympus).

\subsection{4 | Statistics and reproducibility}

The in vitro experiments were repeated at least three times unless stated otherwise. As indicated in the figure legends, all quantitative data are presented as the mean \pm SD or mean \pm SEM of three biologically independent experiments or samples. Statistical analyses were performed using GraphPad Prism 8 and Excel. Statistical significance was tested using an unpaired Student's t test or one/two-way ANOVA with Sidak's multiple-comparisons test. ANOVA was used to compare more than two groups. Exact $P$-values are included in Table S5. All data were considered statistically significant at ${ }^{*} P<.05,{ }^{* *} P<.01$.

\section{3 | RESULTS}

\subsection{YAP is activated in the normal bovine endometrium during early pregnancy}

To gain insight into transcriptional regulation during early pregnancy, we examined RNA-sequencing data ${ }^{25}$ and microarray data from the GEO database (GSE107891). Consistent with previous analyses, ${ }^{25,26}$ genes enriched in gene ontology (GO) terms for cellular component, ECM and cell adhesion were downregulated in pregnant cows compared to non-pregnant cows (Figure S1). In bovines, the uterine endometrium is primarily composed of stromal cells and luminal (marked by EpCAM) and gland epithelial cells (marked by Foxa2) (Figure 1A). In the cohort of samples of bovine endometrium from successful pregnancies ( $n=25)$ and non-pregnancy uteri in oestrus ( $n=10$ ), we confirmed this phenomenon. The results of histopathology experiments showed that the number of glands and the gap between endometrial cells increased in cattle early pregnant (Figure 1A,B). The expression of E-cadherin was markedly decreased in the pregnancy group, indicating weakened cell adhesion junctions (Figure $1 C, D$ ). These results are consistent with the previously reported E-cadherin mediated regulation of epithelial cell polarity loss, ensuring embryo attachment. ${ }^{27}$ Therefore, we speculated that YAP is functionally required for successful pregnancy, and is a sensor and mediator of the structural and mechanical features of the cellular microenvironment. We found that the mRNA and protein levels of YAP were significantly higher in the pregnant group than in the nonpregnant group and that the phosphorylation levels of YAP were significantly decreased (Figure 1D-F). We also assayed two of the best YAP regulated genes from our signature, CTGF and ANKRD1, and the results showed that their expression levels were high in the uterus of pregnant cows (Figure 1G). In addition, we found that YAP was clearly cytoplasmic in the pregnant endometrium but became predominantly nuclear in the non-pregnant endometrium, and YAP expression in endometrial epithelial cells was higher than that in stromal cells (Figure $1 \mathrm{H}, \mathrm{I}$ ). These findings prompted us to investigate whether YAP activation in the endometrium may regulate pregnancy. 


\section{2 | YAP is regulated by cell spreading and ECM} stiffness in bovine endometrial cells

To investigate the cross-talk between YAP and mechanical cues in the bovine endometrium during pregnancy, primary epithelial and stromal cells were isolated according to previously described methods. ${ }^{28}$ The stromal cell marker vimentin and epithelial cell marker keratin 18 (CK18) were used to determine the purity of isolated cells, and when the cell purity was $95 \%$ or higher, the cells were used for further experiments (Figure 2A). A previous
(A)

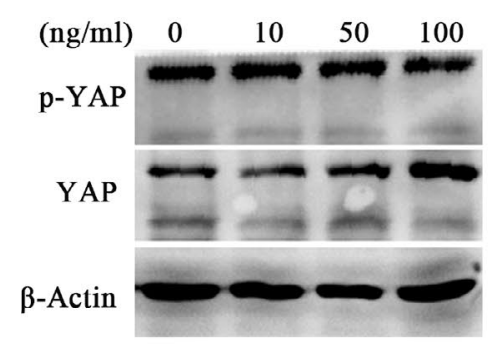

(B)

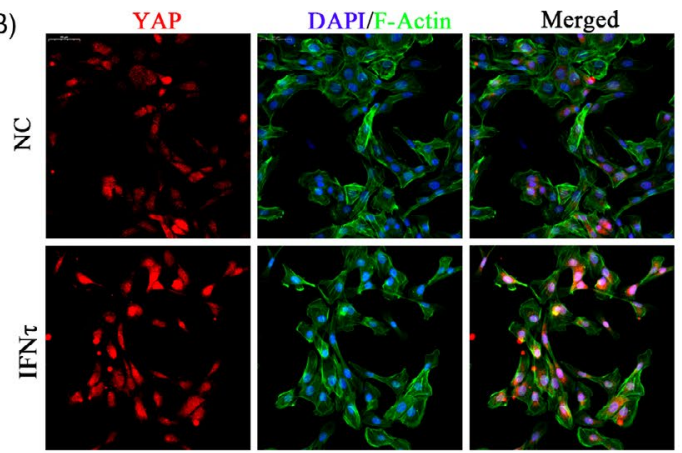

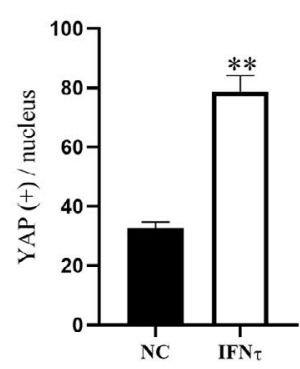

(E)
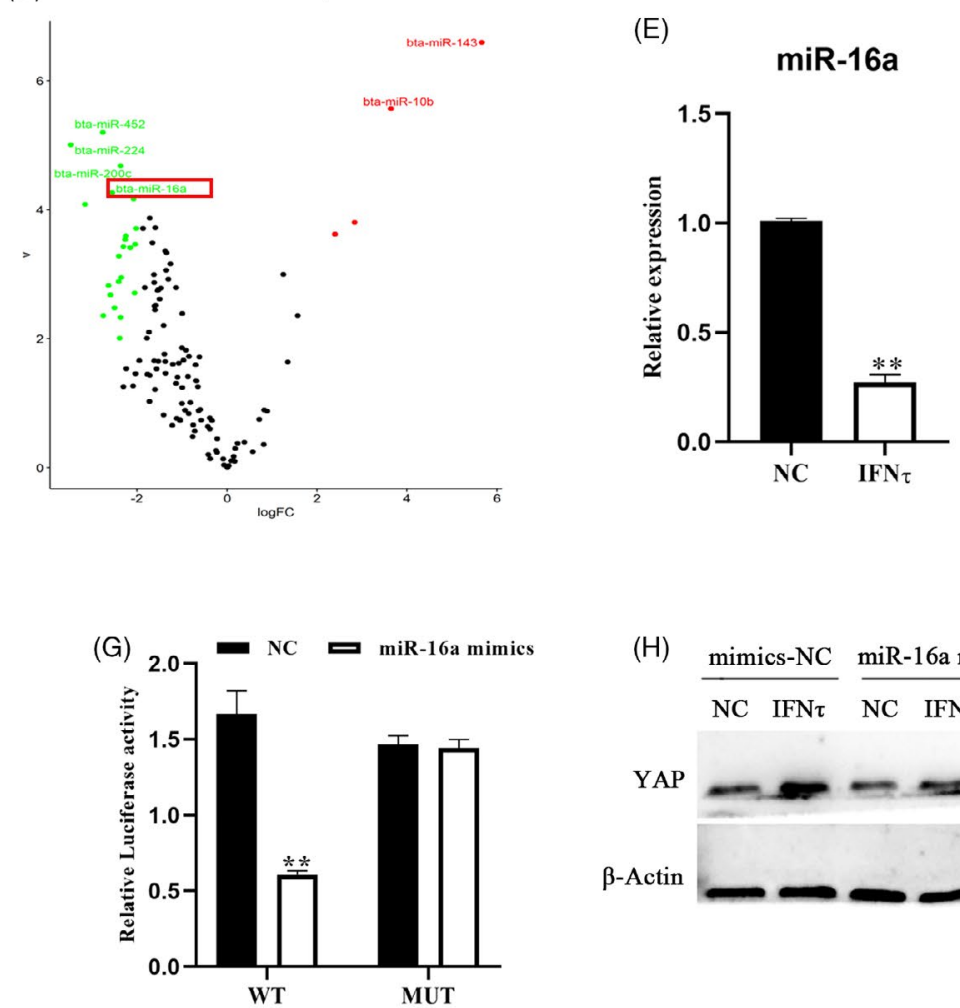

(D) a down stable up

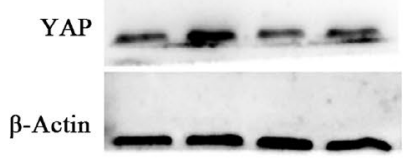

(F)
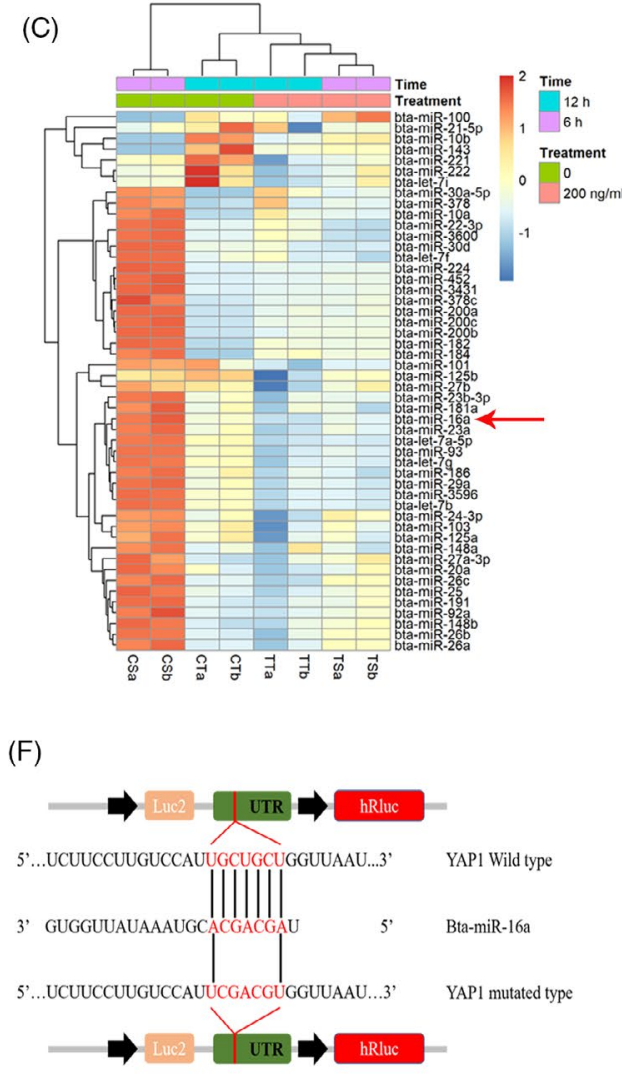

(H) $\frac{\text { mimics-NC }}{\mathrm{NC} \text { IFN } \tau} \frac{\text { miR-16a mimics }}{\mathrm{NC}}$
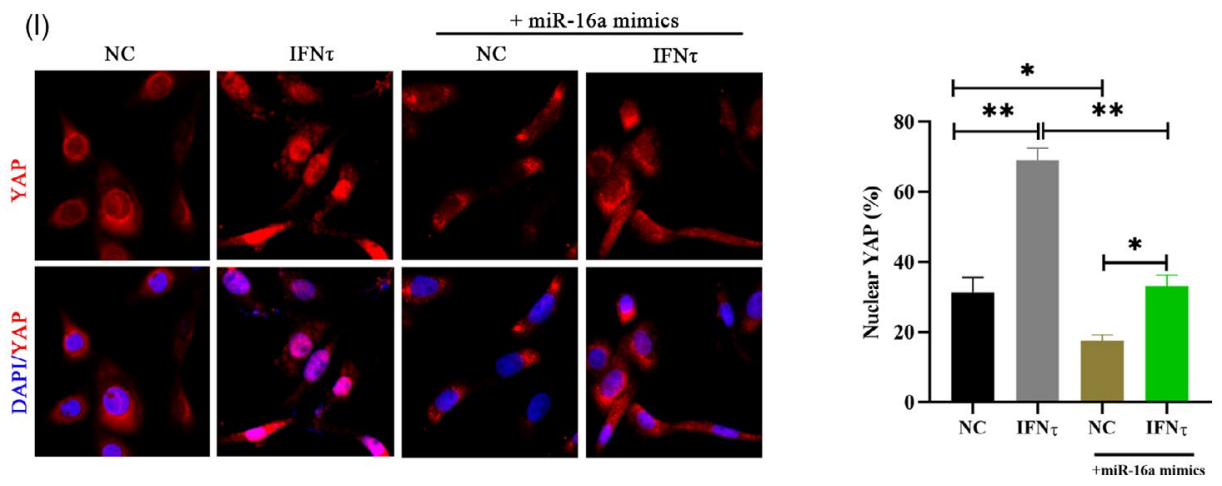

(J)

miR-16a

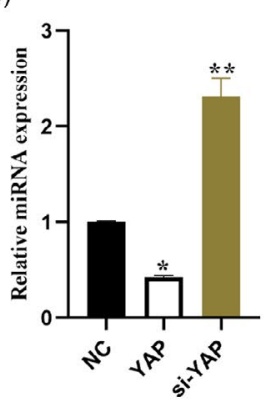


FIGURE 4 IFN $\tau$ mediates the regulation of YAP by decreasing the expression of miR-16a. A, Protein expression levels of YAP detected in bEECs treated with 10,50 , or $100 \mathrm{ng} / \mathrm{ml} \mathrm{IFN} \tau$ for 24 hour by western blot, $\mathrm{n}=3$. B, Immunofluorescence images (left, $\mathrm{n}=2$ ) and quantification of YAP positive cells (right, $\mathrm{n}=5$ ) in bEECs treated with $100 \mathrm{ng} / \mathrm{ml} \mathrm{IFN} \tau$. Scale bars, $50 \mu \mathrm{m}$. C, Heatmap showing commonly expressed miRNAs with significant expression variance. The colour scale indicates relative expression levels of miRNAs. CT and CS: PBS treatment for 12 hour and 6 hour, respectively. TS and TT: IFN $\tau$ treatment for 12 hour and 6 hour, respectively. D, Volcano plot for abnormal expression of miRNAs after 12 hour of bovine epithelial cells treated with $100 \mathrm{ng} / \mathrm{ml} \mathrm{IFN} \tau$. Differentially expressed miRNA were exhibited a 2-fold change in expression with an adjusted $P$ value of 0.05 . E, RT-qPCR analysis of relative miR-16a expression levels in bEECs treated with $100 \mathrm{ng} / \mathrm{ml} \mathrm{IFN} \tau, \mathrm{n}=3$. F, Schematic diagram showing dual-luciferase reporter constructs harbouring the 3'-UTR of YAP with the putative miR-16a-binding site. G, Luciferase activity was measured using the dual-luciferase reporter assay system, $n=3$. $H$, Expression of YAP protein after treatment with the indicated regimen, $n=3$. I, Confocal immunofluorescence images $(n=2)$ and quantification of YAP positive cells (right, $\mathrm{n}=15$ ) in bEECs transfected with mimics-NC or miR-16a mimics and treated with IFN $\tau$ (100 ng/ml) or PBS. J, Expression levels of miR-16a in bEECs transfected with siNC, siYAP and pcDNA3.1(+) YAP were detected using RT-qPCR, $n=3$. Experiments were repeated $n$ times with two biological replicates. Data are shown as the mean \pm SEM. $P$ values were determined by an unpaired two-sided $t$ test (B, E), one-way ANOVA (I, J) and two-way ANOVA (G). ${ }^{*} P<.05,{ }^{* *} P<.01$. See also Figure $\mathrm{S} 3$

study reported that YAP activity is affected by cell density in cancer cells ${ }^{29}$; therefore, we hypothesized that the low cell density of bEECs induced YAP activation. When bEECs were grown at low cell density ( $<40 \%$ confluent), YAP became dephosphorylated and translocated into the nucleus compared to cells grown at high density ( $>80 \%$ confluent) (Figure $2 \mathrm{~B}-\mathrm{E}$ ). We also found that the expression of the YAP target genes CTGF and ANKRD1 was repressed by growth at low density in bEECs (Figure 2F). In addition, cell density obviously affected YAP phosphorylation on serine 127 (Figure 2C), and we speculated that cell density mediated YAP activation was dependent on the Hippo signalling pathway. Then we knocked down LATS1/2, which is a core protein kinase of the Hippo pathway that induces phosphorylation of YAP, by siRNA interference (Figure S2A,B). The results suggested that knockdown of LATS1/2 can rescue the expression of YAP in cells at high confluence (Figure 2G,H, Figure S2C). Thus, cell crowding controls YAP in a manner largely dependent on the Hippo signalling pathway.

To test whether YAP activity is regulated by ECM stiffness in bEECs, we evaluated YAP pathway activity in bEECs grown on fibronectin-coated acrylamide hydrogels of varying stiffness (elastic modulus ranging from 1 to $40 \mathrm{kPa}$, matching the physiological elasticities of natural tissues). The activity of YAP in cells grown on stiff hydrogels $(40 \mathrm{kPa})$ was comparable to that of cells grown at low cell density, whereas growing cells on soft matrices $(\sim 1 \mathrm{kPa})$ inhibited YAP activity to levels comparable to those of cells grown at high cell density (Figure 3A-D, Figure S2D). Interestingly, knockdown of LATS1/2 was not sufficient to block YAP regulation by mechanical forces in in vitro cultured cells grown on synthetic substrates (Figure 3E,F, Figure S2E). The results indicated that YAP regulation through matrix stiffening was largely independent of the Hippo pathway. Based on previous studies ${ }^{30,31}$ and the above results (Figures $2 \mathrm{C}, 3 \mathrm{C}$ ), we also speculated that F-actin integrity is required for YAP to maintain sensitivity to cell density and ECM compliance. In bEECs, activation of YAP by growth at a low-density or on a stiff hydrogel was abolished by the addition of latrunculin A, an F-actin inhibitor (Figure 3G, Figure S2F). Moreover, plating cells at high density combined with soft matrices to inhibit YAP activity (Figure $3 \mathrm{H}$, Figure S2G,H). Collectively, these observations suggest that YAP activity and subcellular localization are regulated by the Hippo signalling pathway and mechanical cues.

\subsection{YAP/TAZ activity is regulated by IFN $\tau$}

In cattle, IFN $\tau$ expression is spatiotemporally specific, and IFN $\tau$ represents an important signalling cytokine during embryo recognition. ${ }^{11}$ Next, we explored whether IFN $\tau$ treatment affects the transcriptional activity of YAP. We assayed two of the most strongly IFN $\tau$ regulated genes, ${ }^{32}$ ISG12 and $M X 2$, by real-time PCR to show the effect of IFN $\tau$ (Figure S3A). Our results demonstrated that both the mRNA and protein levels of YAP increased in the IFN $\tau$ treatment group and that YAP translocated into the nucleus (Figure 4A,B and Figure S3B). Mechanistically, we noticed that IFN $\tau$ did not conspicuously affect YAP phosphorylation compared to that in the control group (Figure 4A), and then we speculated that IFN $\tau$ regulates YAP expression through an abnormally expressed miRNA. Analysing data from our previous study, ${ }^{32}$ we observed that miRNA-16a attenuation by IFN $\tau$ was inversely correlated with the expression levels of YAP. (Figure 4C-E, Figure $\mathrm{S} 3 \mathrm{C}$ ). The results of the double-luciferase reporting experiment showed that compared to the MUT YAP-3'UTR vector groups, luciferase activities were significantly decreased after cotransfection with WT YAP-3'UTR vectors and miR-16a mimics, confirming that YAP is a direct target gene of miR-16a (Figure 4F,G, Figure S3D). Similarly, we also found that miR-16a partially blocked the activation of YAP by IFN $\tau$ (Figure $4 \mathrm{H}, \mathrm{I}$ and Figure S3E-G). Previous studies have reported that the Hippo pathway regulates miRNA biogenesis through nuclear binding of YAP and sequestration of p72 (DDX17) in cancer. ${ }^{33}$ Interestingly, we observed that YAP overexpression reduced miR-16a levels in bEECs (Figure 4J and Figure $\mathrm{S} 3 \mathrm{H}$ ). Physiologically, this positive feedback mechanism allows amplification of embryo-derived signals. Therefore, we suggest that bEECs may respond to IFN $\tau$ signals released from the conceptus by activating the miR-16a/YAP axis. 
(A)

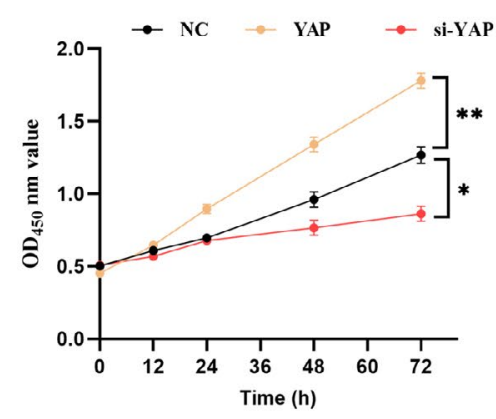

(C)

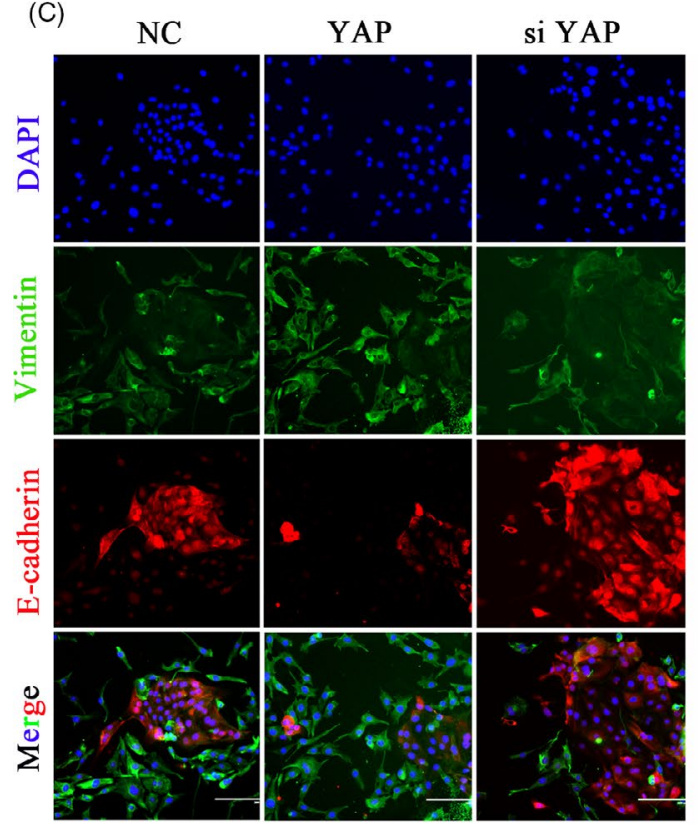

(B)
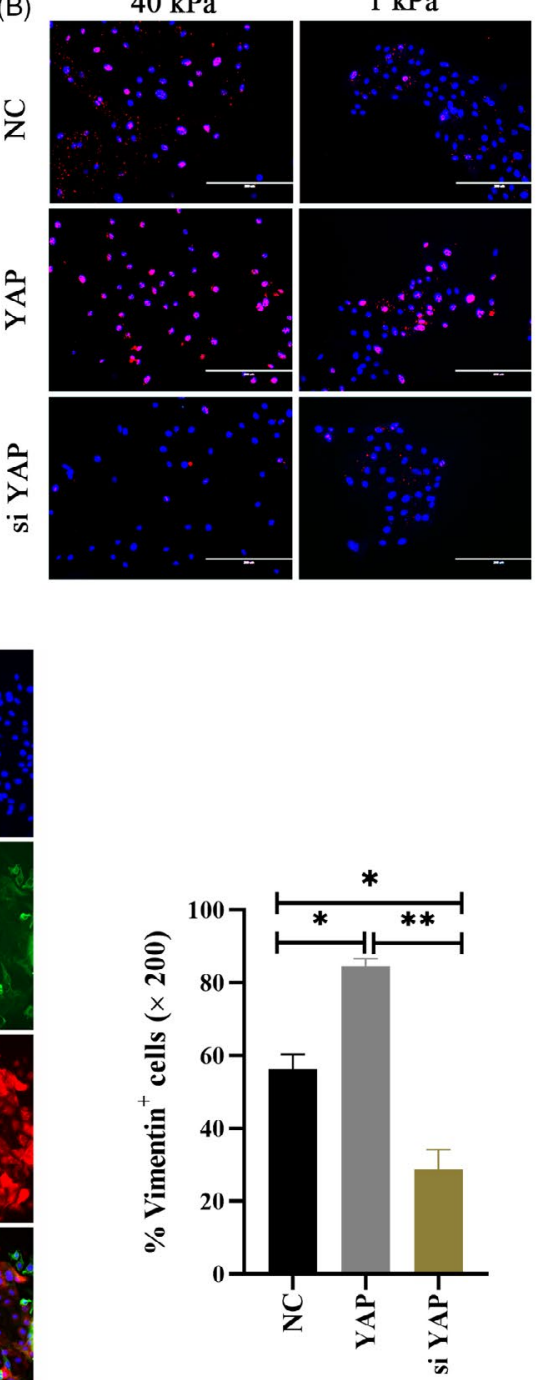

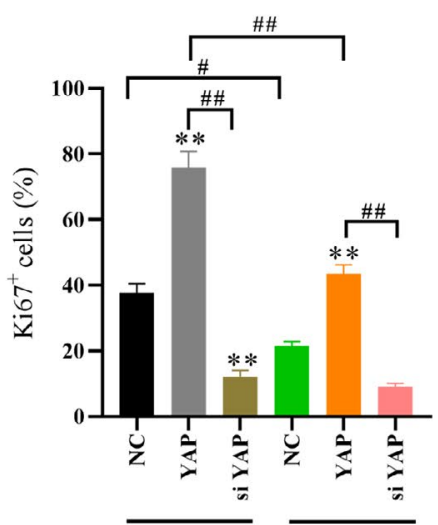

$40 \mathrm{kPa}$

$1 \mathrm{kPa}$
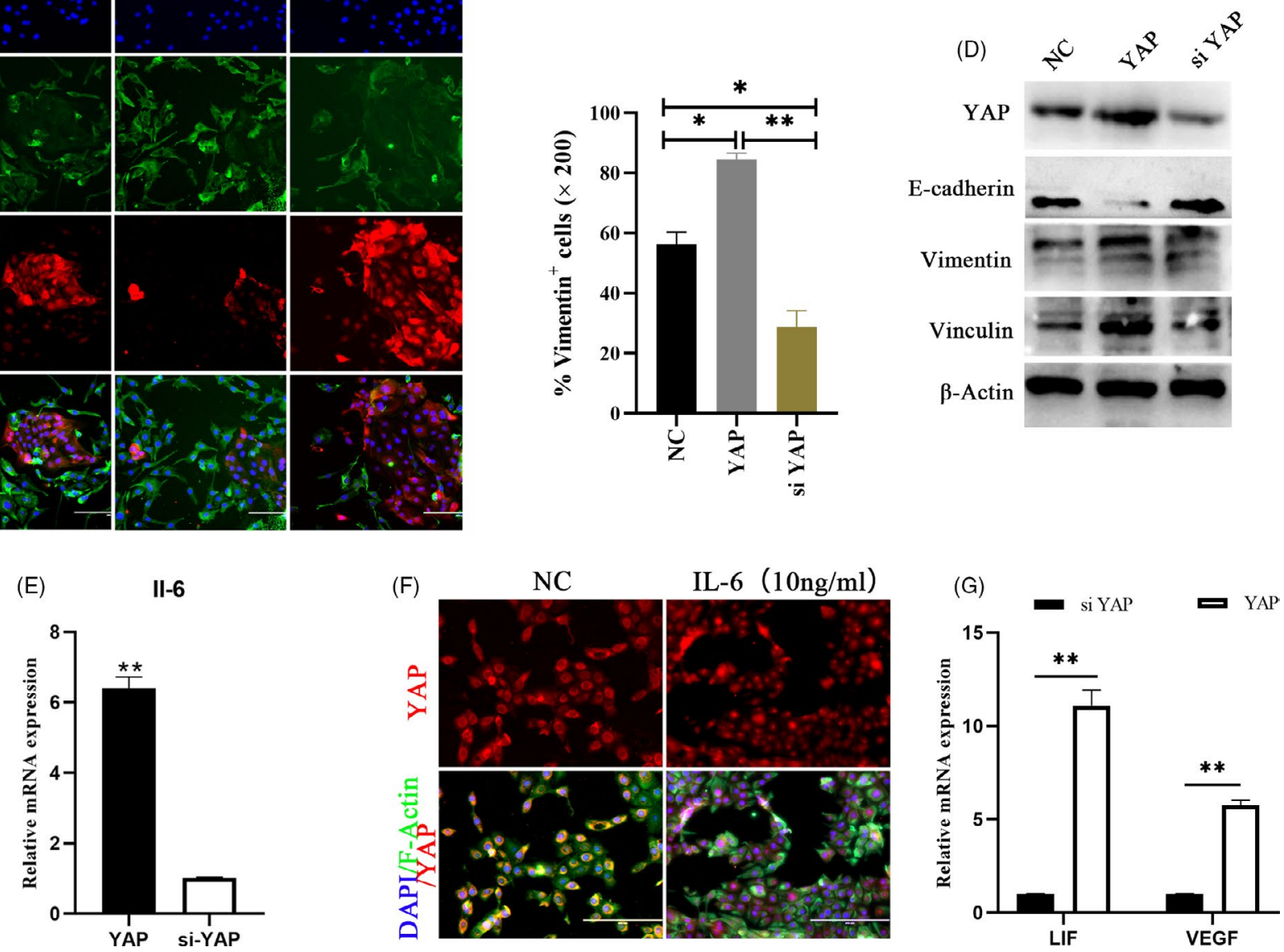

FIGURE 5 YAP activation can provides the physiological environment needed for early pregnancy. A, CCK-8 kits were used to assess proliferation of bEECs transfected with siNC, siYAP or pcDNA3.1(+) YAP at 0, 12, 24, 48 and 72 hour. ${ }^{*} P<.05$, ${ }^{* *} P<.01$. B, Immunofluorescence images (left) and quantifications of Ki67positive cells (right) in bEECs transfected with siNC, siYAP or pcDNA3.1(+) YAP at $40 \mathrm{kPa} / 1 \mathrm{kPa}$ hydrogels, $\mathrm{n}=3$. Scale bars, $200 \mu \mathrm{m} .{ }^{* *} P<.01,{ }^{\#} P<.05,{ }^{\# \#} P<.01 . \mathrm{C}$, Immunofluorescence images of $\mathrm{E}-\mathrm{cadh}$. vimentin (left, $n=2$ ) and quantification of vimentin ${ }^{+}$positive cells (right, $n=5$ ) in bEECs transfected with YAP overexpression plasmid or siYAP at 48 hour. Scale bars, $200 \mu \mathrm{m}$. E-cadherin, red; DAPI, blue; Vimentin, green. ${ }^{*} P<.05,{ }^{* *} P<.01$. D, Protein expression levels of YAP, E-cadherin, vimentin and vinculin were detected in bEECs, $n=3$. E, RT-qPCR analysis of relative IL- 6 expression levels in bEECs transfected with siYAP or pcDNA3.1(+) YAP, $n=3$. F, Representative immunofluorescence of YAP in bEECs treated with IL-6 (10 ng/ml) or PBS at 24 hour, $n=2$. G, RT-qPCR analysis of relative LIF and VEGF expression levels in bEECs transfected with siYAP or pcDNA3.1(+) YAP, $n=3$. ${ }^{* *} P<.01$. Experiments were repeated $n$ times with two biological replicates. Data are shown as the mean \pm SEM. $P$ values were determined by an unpaired two-sided t test $(e, g)$ and two-way ANOVA $(A-C)$. See also Figure S4 


\section{4 | YAP activation provides the physiological environment needed for early pregnancy}

Unsurprisingly, as in other cell types, ${ }^{17}$ YAP activation induced by mechanical or chemical signals promoted proliferation of bEECs (Figure 5A,B). This provides a theoretical basis for the growth and replacement of the maternal endometrium during pregnancy. Furthermore, we observed a decrease in E-cadherin expression and an increase in vimentin activation in cells overexpressing YAP (Figure 5C,D, Figure S4A). This result indicates that switch-like regulation of YAP activated by mechanical or chemical signals, induces epithelial-mesenchymal transition (EMT) and loosens cell-cell adhesion. Healthy pregnancy requires tightly coordinated immune responses. In early pregnancy, blastocysts attach to and invade the maternal endometrium and this progression is accompanied by evolutionarily conserved inflammatory responses, including IL-6, IL-1, and LIF. ${ }^{34}$ We observed that mechanical or chemical stimulation upregulated YAP induced IL-6 production in bEECs and had no obvious effect on other inflammatory factors (Figure 5E, Figure S4B), which are proposed to be essential for the formation of the maternal uterine immune environment during the preimplantation period. We also found that IL-6 positively regulates YAP activation in bEEc cells (Figure 5F), consistent with previous studies in an organ injury model. ${ }^{35,36}$ The expression of the uterine receptive markers LIF and VEGF also exhibited a positive correlation with YAP (Figure 5G). These results indicate that YAP activation provides the conditions required for early pregnancy.

\section{5 | YAP activation is required for early pregnancy in the mouse endometrium}

To further confirm the activation of YAP during pregnancy, we used a mouse pregnancy model in vivo. In agreement with the bovine in vivo data, the expression of YAP associated genes, including YAP at the protein level and CTGF and ANKRD1 at the mRNA level, was significantly augmented in the mouse model of early pregnancy (Figure 6A-E). These results also revealed that YAP is activated before embryo implantation and without direct mechanotransduction between the embryo and maternal uterus, which indicates that chemical stimulation signals initiate YAP activation during early pregnancy (Figure 6C,D). In addition, we found that intrauterine injection with IFN $\tau$ significantly increased YAP expression in the IFN $\tau$-treated uterus compared to PBS-treated control mice (Figure S5A-E). We also observed that the expression of YAP was highest at the attachment site, which prompted us to speculate that it plays a role in mechanotransduction. Next, we tested whether blocking embryo-derived signals would interfere with YAP activation in vivo using an oil-induced mouse pseudopregnancy model (Figure 6F-H, Figure S5F). Remarkably, the mechanical signals that the cell received from the uterine cavity induced activation of YAP (Figure 6I, Figure S5G). These data suggest that activated YAP is subject to regulation by both mechanical and chemical signal during early pregnancy.

\subsection{YAP activation is necessary for early pregnancy}

To investigate the role of YAP in the maternal uterus during early pregnancy in vivo, we administered the YAP inhibitor verteporfin to pregnant mice (Figure 7A). By western blot analysis, we clearly observed that verteporfin significantly reduced the expression of YAP (Figure 7B). Intraperitoneal injection with verteporfin reduced in the number of implanted embryos in verteporfin-treated uterine horns compared to vehicle-treated control horns (Figure 7C,D). Next, we analysed endometrial cell proliferation after VP treatment with bromodeoxyuridine (BrdU) and YAP double staining in mouse uterine tissue (Figure 7E). By scoring the ratio of $\mathrm{BrdU}^{+}$cells versus total cells, we found that endometrial cell proliferation was decreased in the VP treatment group (Figure 7F). The expression of two implantation markers, HOXA10 and LIF, was also dramatically reduced when YAP was inhibited or knocked down (Figure 7G). In addition, inactivation of YAP leads to a reduction in the inflammatory factor IL-6 that is required for early pregnancy (Figure 7G). To exclude a possible non-specific effect of the YAP inhibitor on pregnant mice and embryos, we inactivated YAP by injecting independent sets of siRNAs into the uterine cavities of mice on ED 3.5 (Figure 7A and H). Similar to verteporfin, siYAP also significantly reduced the number of implanted embryos compared to that of the control treated with negative control siRNAs (Figure $7 \mathrm{H}$ $\mathrm{K})$. Collectively, these results suggest that YAP is a vital factor in the maternal uterus that is necessary for successful pregnancy.

\section{4 | DISCUSSION}

Previous studies of YAP have shown that it determines organ size and tumorigenesis, but investigation of YAP function in the uterus during early pregnancy are limited. Here we show that activation of YAP provides a suitable environment for early pregnancy in maternal endometrial tissue. Indeed, high YAP expression promoted endometrial cell proliferation, induced EMT progression and generated a small inflammatory benefit to enable the uterus to receive the embryo. Mechanistically, the expression levels of YAP are dually regulated by upstream biochemical and mechanical signals in bEECs and are partially dependent on the Hippo signalling pathway.

Although many clues have been uncovered indicating that YAP and TAZ are sensors of the structural and mechanical features of the cellular microenvironment, ${ }^{37,38}$ the exact mechanism by which mechanical signals modulate YAP nuclear localization is not entirely clear. Mechanical inputs represent a central pillar in the control of YAP/TAZ activity, including modification of the actomyosin cytoskeleton, cell polarity, matrix stiffness and cell shape. ${ }^{29,30,39}$ In this study, we confirmed that cell density and ECM stiffness affect YAP expression and nuclear translocation, consistent with previous studies. ${ }^{29,30,39}$ However, there is controversy about whether mechanical signals regulate YAP/TAZ through the Hippo pathway. Recently, some studies have suggested that YAP regulation by stiffness and Rho is independent of the Hippo pathway, ${ }^{39,40}$ but on the other hand, studies 


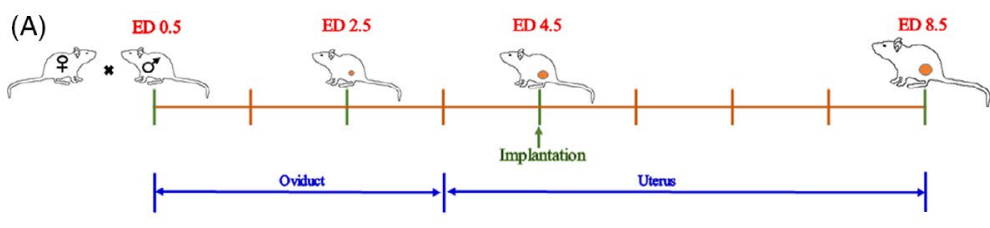

(B)
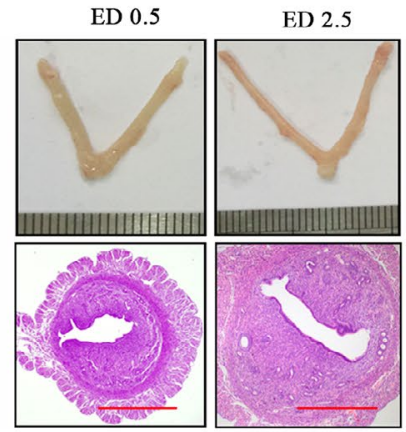
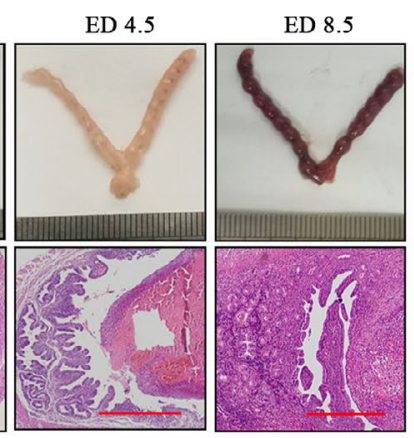

(C)
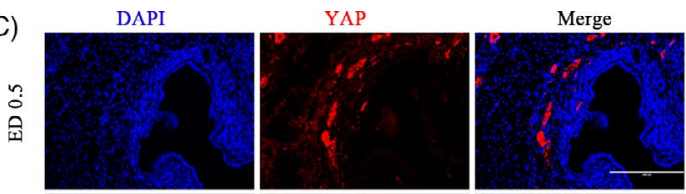

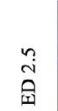
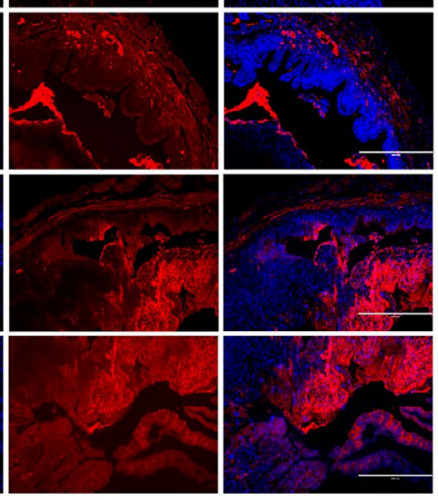

(D)

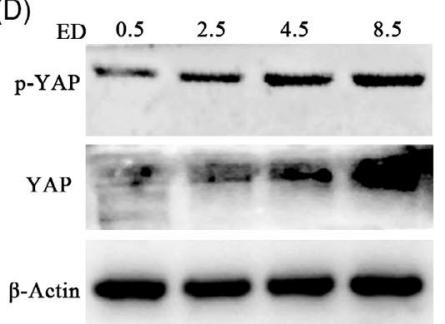

(E)
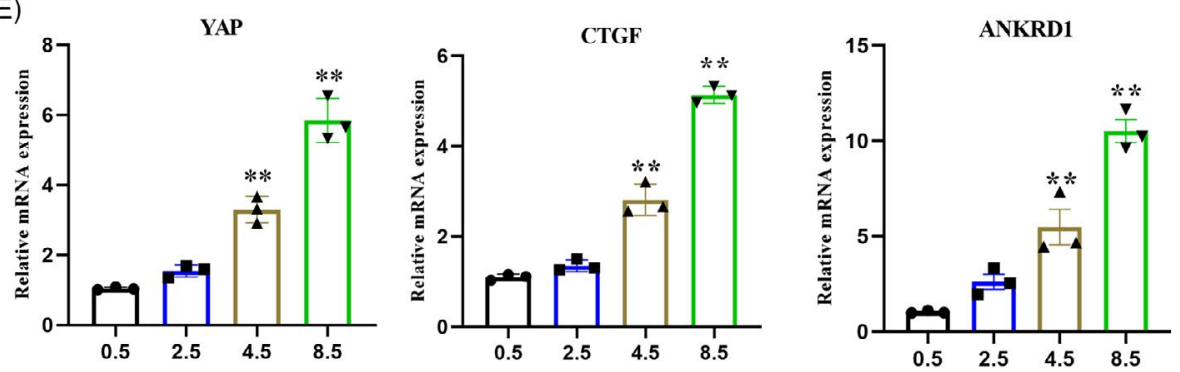

(F)

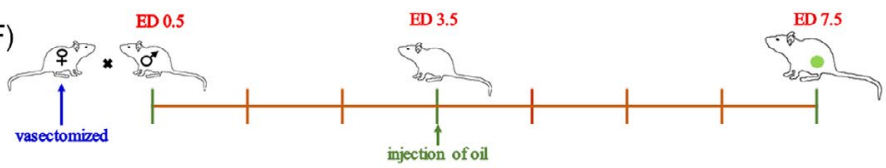

(G)

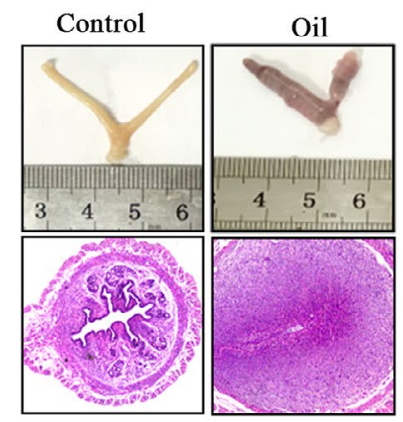

(H)

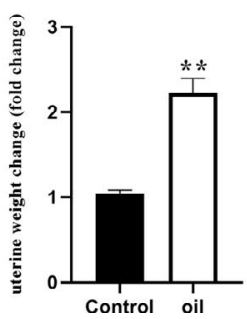

(I)

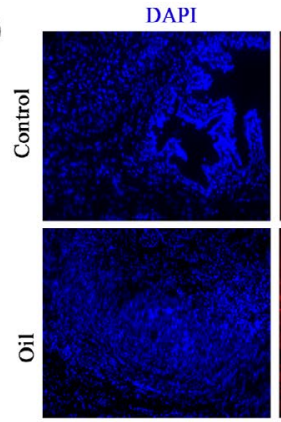

YAP
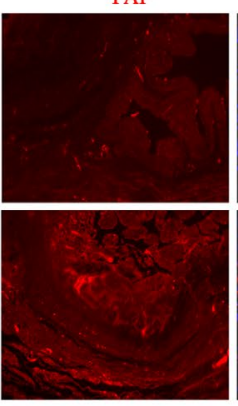

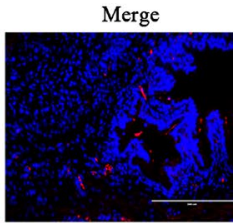

FIGURE 6 YAP is activated by mechanical and chemical cues in mouse pregnancy-related models. A, Timeline of embryo development and euthanasia. B, Whole uterus images and uterine sections stained with H\&E. Scale bars, $500 \mu \mathrm{m}$. C, Immunofluorescence detection of YAP endometrial sections from pregnant mice. Scale bars, $400 \mu \mathrm{m}, \mathrm{n}=2$. D, Protein expression levels of YAP and $p$-YAP were detected in mouse uterine tissue, $n=3$. E, RT-qPCR analysis of YAP, CTGF and ANKRD1 expression levels, $n=3$. F, Schematic outline of the establishment of pseudopregnancy mouse model. G, Schematic illustration of pseudopregnant mouse model. Whole uterus image and uterus sections stained with H\&E. Scale bars, $500 \mu \mathrm{m}$. H, Effect of oil on uterine weight change $4 \mathrm{~d}$. Data are shown as the fold change compared to controls without oil injection, $n=6$. I, Immunofluorescence images of YAP showing uterine cross-sections from pseudopregnant mice $4 \mathrm{~d}$ after intrauterine injection of oil, $\mathrm{n}=2$. Experiments were repeated $\mathrm{n}$ times with two biological replicates. Data are presented as the mean \pm SEM. $P$ values were determined by unpaired two-sided $t$ tests $(h)$ and one-way ANOVA (e). ${ }^{*} P<.05,{ }^{* *} P<.01$. See also Figure $S 5$

have demonstrated that YAP regulation by matrix stiffening involves both the actin cytoskeleton and the Hippo pathway. ${ }^{30,41}$ Here, we showed that the effect of cell density is largely dependent on the
Hippo pathway, and that the ECM yielded the opposite results. This inconsistency could be due to the form, character or magnitude of the mechanical forces, and the threshold difference needed for cells 
(A)

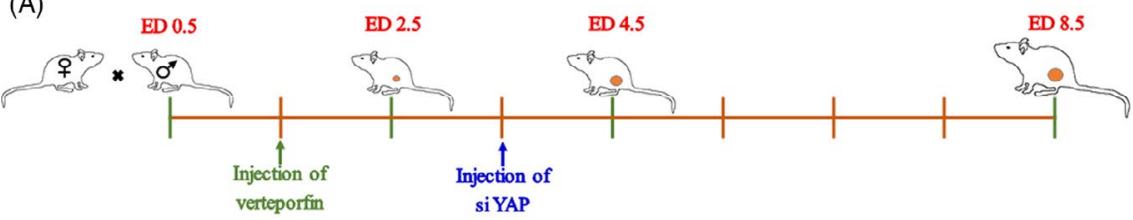

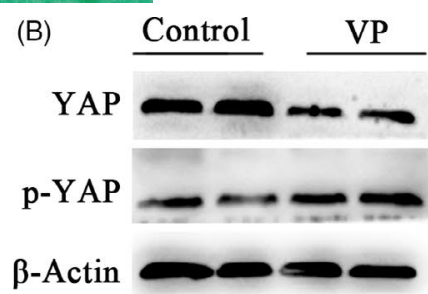

(C) (E) DAPI YAP
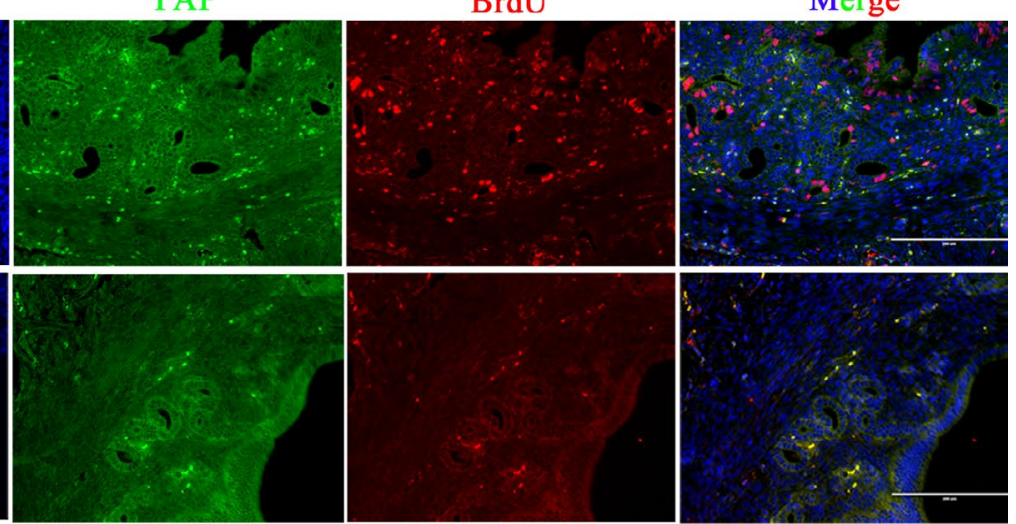

(D)

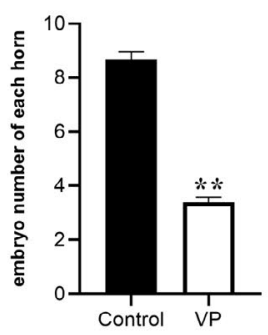

(H)
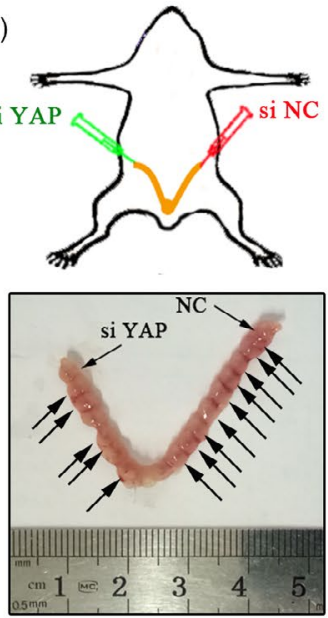

(F)

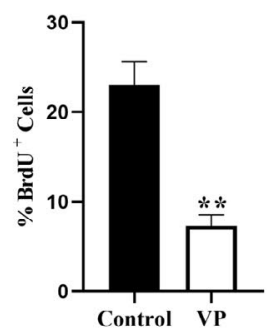

(I)

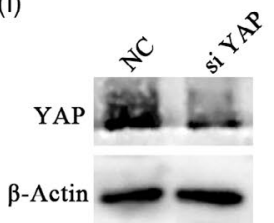

(J)

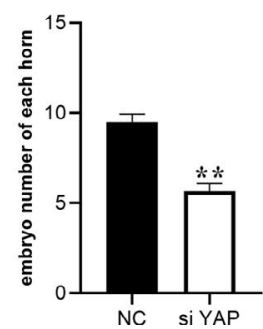

(G)

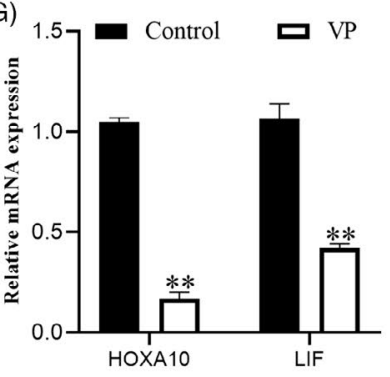

(K)
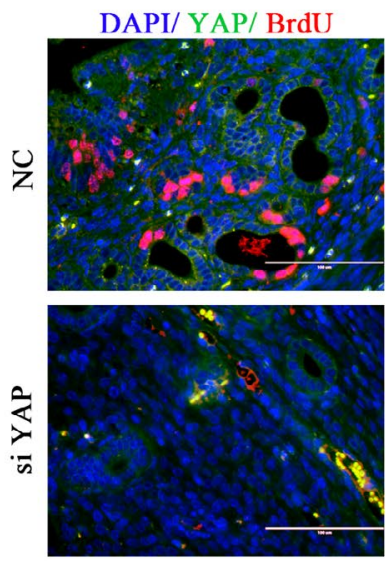
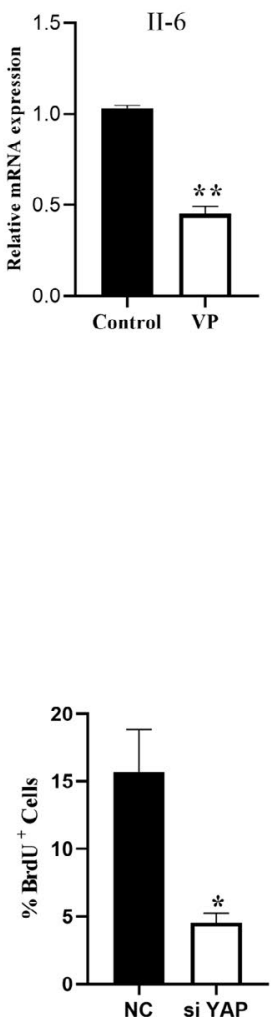

FIGURE 7 YAP is required for embryo implantation in vivo. A, Schematic illustration of intrauterine injection of siYAP or intraperitoneal injection verteporfin. B, Protein expression levels of YAP and $\mathrm{p}$-YAP were detected in mouse treated with VP or PBS (control), $\mathrm{n}=3$. C, Representative image of embryo implantation sites from the control and VP groups at ED 8.5. D, The number of implanted embryos observed in each uterine horn on day ED 8.5. Control, $n=14 ; \mathrm{VP}, \mathrm{n}=9$. E, F, Costaining of BrdU with YAP (e) and quantification of BrdU ${ }^{+}$ positive cells ( $f$ ) in mouse endometrial sections, $n=2$. Scale bars, $200 \mu \mathrm{m}$. G, RT- qPCR analysis of mRNA levels of IL-6, HOXA 10 and LIF in the mouse uterus, $n=3$. H, Effect of YAP knockdown on implantation rate in mice with an image showing implantation sites (arrows) in the control uterine horn (right, siNC) compared to the siYAP treated samples. I, Western blotting data showing uterine YAP levels after injection of siNC or siYAP, $n=3$. J, The number of implanted embryos observed in each uterine horn on day ED 8.5. $n=6$. K, Costaining of BrdU with YAP and quantification of positive cells (right) of $\mathrm{BrdU}^{+}$in mouse endometrial sections, $\mathrm{n}=2$. Scale bars, $100 \mu \mathrm{m}$. Experiments were repeated $n$ times with two biological replicates. Data are mean \pm SEM. $P$ values were determined by an unpaired two-sided $t$ test $(D, E, I)$ and one-way ANOVA (F). ${ }^{* *} P<.01$ 
to detect the mechanical force. In addition, there is a consensus that actin is important for mechanotransduction, and the Hippo pathway contributes to remodelling both the actin cytoskeleton and the ECM. ${ }^{42}$

Quite a few types of soluble factors have been reported to activate YAP in mammals; these factors include LPA and oestrogen, which depend on $\mathrm{G}$ protein-coupled receptors (GPCRs). ${ }^{43,44}$ In contrast, cytokines, insulin and IL-6 act independently of GPCRs and can also modulate cell processes via the Hippo-YAP pathway. ${ }^{38,45}$ Our study suggests that IFN $\tau$ is a critical factor for pregnancy recognition in ruminant ungulates and activates YAP in bEECs. Moreover, we revealed that miRNA-16a is the core downstream component of IFN $\tau$-mediated YAP activation. It has been reported that YAP represses miRNA biogenesis and may be involved in the widespread miRNA repression observed in cancer. ${ }^{33}$ This indirectly explains why we observed that YAP inhibits expression of miRNA-16a. Interestingly, an early study demonstrated that YAP regulates IFN $\tau$ expression via the TEAD transcription factor in bovine conceptuses. ${ }^{46}$ Taken together, this seems to imply that there is a complex feedback network between IFN $\tau$ and YAP during early pregnancy. Future work will need to focus on revealing the relative contribution and possible collaboration of biochemical and mechanical signals between the embryo and the uterus.

Although YAP appears to be dispensable for physiological homoeostasis in several adult organs, it is critical to promote tissue repair in response to injury and serves as an oncogene in many types of tumours. ${ }^{37,45}$ Here, we demonstrated that YAP activity is regulated by both biochemical and mechanical cues and that its activation promotes endometrial cell proliferation. Consistent with previous studies, YAP is highly expressed in cancer and correlated with cancer cell characteristics, such as proliferation, migration and apoptosis. ${ }^{47,48}$ Although YAP contribute to repair and alleviates inflammatory damage, ${ }^{15,49}$ we showed that YAP induced the production of the proinflammatory cytokine IL-6. In embryos, differences in YAP expression levels and subcellular localization between internal and external cell populations lead to changes in cell fate specification during trophectoderm (TE) formation. ${ }^{50}$ Outer TE cells invade the endometrium and are involved in the EMT process, and our data demonstrate that overexpression of YAP upregulates of EMT-associated transcription factor expression in bEECs. Furthermore, we used mouse pregnancy and pseudopregnancy models to reveal a crucial role for YAP activation by mechanical and chemical signals in facilitating successful pregnancy. Inhibiting the activation of YAP causes the failure of conceptus attachment to the uterine epithelium and a reduction in the number of embryos.

In summary, this study is the first to show that activation of YAP is necessary for early pregnancy in bovines because it induces the maternal uterus to provide an environment suitable for conceptus survival. Mechanistically, we suggest that YAP is a sensor for endometrial cells to respond to extracellular chemical and mechanical signals, and that different stimuli converge on YAP regulation through the IFN $\tau /$ miR-16a/YAP or Hippo/F-Actin/YAP pathways. Identification of this underlying mechanistic axis provides novel insights into how the maternal uterus responds to signals from the surrounding environment during early pregnancy.

\section{ACKNOWLEDGEMENTS}

We thank all members of the Laboratory of Veterinary Clinical Diagnosis for helpful discussions and suggestions.

\section{CONFLICT OF INTERESTS}

The authors declare no competing financial interests.

\section{AUTHOR CONTRIBUTIONS}

TZ and GD conceived and designed the experiments. TZ, SG, and $\mathrm{HZ}$ carried out the experiments. $\mathrm{TZ}, \mathrm{CQ}$, and $\mathrm{GZ}$ analysed the data. TZ, ZW, J.L, and GD wrote the manuscript. All authors agreed to be responsible for the content of the work.

\section{DATA AVAILABILITY STATEMENT}

All other data that support the findings of this study are available on request from the corresponding author. The RNA-seq datasets analysed in this article are publicly available in the GEO database under the identifiers GSE107891, https://doi.org/10.3168/jds.2011-5114 and https://doi.org/10.18632/oncotarget.18470.

\section{ORCID}

Changwei Qiu iD https://orcid.org/0000-0002-7389-9534

Ganzhen Deng (iD https://orcid.org/0000-0003-0813-927X

\section{REFERENCES}

1. Chapron C, Marcellin L, Borghese B, Santulli P. Rethinking mechanisms, diagnosis and management of endometriosis. Nat Rev Endocrinol. 2019;15(11):666-682.

2. Zhang $H$, Wang $S$, Zhang $S$, Wang $T$, Deng $X$. Increasing trend of prevalence of infertility in Beijing. Chin Med J (Engl). 2014;127(4):691-695.

3. Yuan M, Hu M, Lou Y, et al. Environmentally relevant levels of bisphenol $\mathrm{A}$ affect uterine decidualization and embryo implantation through the estrogen receptor/serum and glucocorticoid-regulated kinase 1/epithelial sodium ion channel alpha-subunit pathway in a mouse model. Fertil Steril. 2018;109(4):735-744.e731.

4. $\mathrm{MaH}$, Zhai J, Wan $\mathrm{H}$, et al. In vitro culture of cynomolgus monkey embryos beyond early gastrulation. Science. 2019;366(6467):eaax7890.

5. Niu Y, Sun N, Li C, et al. Dissecting primate early post-implantation development using long-term in vitro embryo culture. Science. 2019;366(6467):eaaw5754.

6. Ruan YC, Guo JH, Liu X, et al. Activation of the epithelial Na+ channel triggers prostaglandin $E(2)$ release and production required for embryo implantation. Nat Med. 2012;18(7):1112-1117.

7. Ramathal CY, Bagchi IC, Taylor RN, Bagchi MK. Endometrial decidualization: of mice and men. Semin Reprod Med. 2010;28(1):17-26.

8. Salker MS, Christian M, Steel JH, et al. Deregulation of the serumand glucocorticoid-inducible kinase SGK1 in the endometrium causes reproductive failure. Nat Med. 2011;17(11):1509-1513.

9. Turco MY, Gardner L, Kay RG, et al. Trophoblast organoids as a model for maternal-fetal interactions during human placentation. Nature. 2018;564(7735):263-267.

10. Roberts RM, Cross JC, Leaman DW. Interferons as hormones of pregnancy. Endocr Rev. 1992;13(3):432-452.

11. Roberts RM. Interferon-tau, a Type 1 interferon involved in maternal recognition of pregnancy. Cytokine Growth Factor Rev. 2007;18(5-6):403-408.

12. Jayyosi C, Lee $\mathrm{N}$, Willcockson A, Nallasamy S, Mahendroo $\mathrm{M}$, Myers $\mathrm{K}$. The mechanical response of the mouse cervix to 
tensile cyclic loading in term and preterm pregnancy. Acta Biomater. 2018;78:308-319.

13. Grund S, Grummer R. Direct cell(-)cell interactions in the endometrium and in endometrial pathophysiology. Int J Mol Sci. 2018;19(8).

14. Schatz F, Guzeloglu-Kayisli O, Arlier S, Kayisli UA, Lockwood CJ. The role of decidual cells in uterine hemostasis, menstruation, inflammation, adverse pregnancy outcomes and abnormal uterine bleeding. Hum Reprod Update. 2016;22(4):497-515.

15. Li C, Jin Y, Wei S, et al. Hippo signaling controls NLR family pyrin domain containing 3 activation and governs immunoregulation of mesenchymal stem cells in mouse liver injury. Hepatology. 2019;70(5):1714-1731.

16. Zhao B, Tumaneng K, Guan KL. The Hippo pathway in organ size control, tissue regeneration and stem cell self-renewal. Nat Cell Biol. 2011;13(8):877-883.

17. Yu FX, Zhao B, Guan KL. Hippo pathway in organ size control, tissue homeostasis, and cancer. Cell. 2015;163(4):811-828.

18. Sun S, Irvine KD. Cellular organization and cytoskeletal regulation of the hippo signaling network. Trends Cell Biol. 2016;26(9):694-704.

19. Park JA, Kwon YG. Hippo-YAP/TAZ signaling in angiogenesis. BMB Rep. 2018;51(3):157-162.

20. Mammoto T, Ingber DE. Mechanical control of tissue and organ development. Development. 2010;137(9):1407-1420.

21. Zhou F, Wang R, Yuan $P$, et al. Reconstituting the transcriptome and DNA methylome landscapes of human implantation. Nature. 2019:572(7771):660-664.

22. Peng G, Suo S, Cui G, et al. Molecular architecture of lineage allocation and tissue organization in early mouse embryo. Nature. 2019;572(7770):528-532

23. Cronin JG, Kanamarlapudi V, Thornton CA, Sheldon IM. Signal transducer and activator of transcription-3 licenses Toll-like receptor 4-dependent interleukin (IL)-6 and IL-8 production via IL-6 receptor-positive feedback in endometrial cells. Mucosal Immunol. 2016;9(5):1125-1136

24. Tse JR, Engler AJ. Preparation of hydrogel substrates with tunable mechanical properties. Curr Protoc Cell Biol. 2010;10:Unit 10.16.

25. Cerri R, Thompson IM, Kim IH, et al. Effects of lactation and pregnancy on gene expression of endometrium of Holstein cows at day 17 of the estrous cycle or pregnancy. J Dairy Sci. 2012;95(10):5657-5675.

26. Moraes JGN, Behura SK, Geary TW, Hansen PJ, Neibergs HL, Spencer TE. Uterine influences on conceptus development in fertility-classified animals. Proc Natl Acad Sci USA. 2018;115(8):E1749 -E1758.

27. Daikoku T, Cha J, Sun X, et al. Conditional deletion of Msx homeobox genes in the uterus inhibits blastocyst implantation by altering uterine receptivity. Dev Cell. 2011;21(6):1014-1025.

28. Turner ML, Cronin JG, Healey GD, Sheldon IM. Epithelial and stromal cells of bovine endometrium have roles in innate immunity and initiate inflammatory responses to bacterial lipopeptides in vitro via Toll-like receptors TLR2, TLR1, and TLR6. Endocrinology. 2014;155(4):1453-1465.

29. Yang $\mathrm{W}-\mathrm{H}$, Ding $\mathrm{C}-\mathrm{K}$, Sun $\mathrm{T}$, et al. The hippo pathway effector TAZ regulates ferroptosis in renal cell carcinoma. Cell Rep. 2019;28(10):2501-2508.e2504.

30. Meng Z, Qiu Y, Lin KC, et al. RAP2 mediates mechanoresponses of the Hippo pathway. Nature. 2018;560(7720):655-660.

31. Dupont S, Morsut L, Aragona M, et al. Role of YAP/TAZ in mechanotransduction. Nature. 2011;474(7350):179-183.

32. Wu H, Zhang T, Ma X, et al. Specific microRNA library of IFN-tau on bovine endometrial epithelial cells. Oncotarget. 2017;8(37):61487-61498.

33. Mori M, Triboulet R, Mohseni M, et al. Hippo signaling regulates microprocessor and links cell-density-dependent miRNA biogenesis to cancer. Cell. 2014;156(5):893-906.
34. Yockey LJ, Iwasaki A. Interferons and proinflammatory cytokines in pregnancy and fetal development. Immunity. 2018;49(3):397-412.

35. Zhou X, Li W, Wang S, et al. YAP aggravates inflammatory bowel disease by regulating M1/M2 macrophage polarization and gut microbial homeostasis. Cell Rep. 2019;27(4):1176-1189.e1175.

36. Li Y, Hu Q, Li C, et al. PTEN-induced partial epithelial-mesenchymal transition drives diabetic kidney disease. J Clin Investig. 2019;129(3):1129-1151.

37. Zanconato F, Cordenonsi M, Piccolo S. YAP/TAZ at the roots of cancer. Cancer Cell. 2016;29(6):783-803.

38. Ma S, Meng Z, Chen R, Guan KL. The hippo pathway: biology and pathophysiology. Annu Rev Biochem. 2019;88:577-604.

39. Hao J, Zhang Y, Wang Y, et al. Role of extracellular matrix and YAP/ TAZ in cell fate determination. Cell Signal. 2014;26(2):186-191.

40. Aragona M, Panciera T, Manfrin A, et al. A mechanical checkpoint controls multicellular growth through YAP/TAZ regulation by actin-processing factors. Cell. 2013;154(5):1047-1059.

41. Wada K, Itoga K, Okano T, Yonemura S, Sasaki H. Hippo pathway regulation by cell morphology and stress fibers. Development. 2011;138(18):3907-3914

42. Bertero T, Cottrill K, Lu YU, et al. Matrix remodeling promotes pulmonary hypertension through feedback mechanoactivation of the YAP/TAZ-miR-130/301 circuit. Cell Rep. 2015;13(5):1016-1032.

43. Zhou $X$, Wang $S$, Wang $Z$, et al. Estrogen regulates Hippo signaling via GPER in breast cancer. J Clin Invest. 2015;125(5):2123-2135.

44. Yu F-X, Zhao B, Panupinthu N, et al. Regulation of the HippoYAP pathway by G-protein-coupled receptor signaling. Cell. 2012;150(4):780-791

45. Taniguchi K, Wu L-W, Grivennikov SI, et al. A gp130-Src-YAP module links inflammation to epithelial regeneration. Nature. 2015;519(7541):57-62.

46. Kusama K, Bai R, Sakurai T, et al. A transcriptional cofactor YAP regulates IFNT expression via transcription factor TEAD in bovine conceptuses. Domest Anim Endocrinol. 2016;57:21-30.

47. Gao Y, Zhang W, Han X, et al. YAP inhibits squamous transdifferentiation of Lkb1-deficient lung adenocarcinoma through ZEB2dependent DNp63 repression. Nat Commun. 2014;5:4629.

48. Murakami S, Shahbazian D, Surana R, et al. Yes-associated protein mediates immune reprogramming in pancreatic ductal adenocarcinoma. Oncogene. 2017;36(9):1232-1244.

49. Liu B, Zheng Y, Yin F, Yu J, Silverman N, Pan D. Toll receptor-mediated hippo signaling controls innate immunity in Drosophila. Cell. 2016:164(3):406-419.

50. Nishioka N, Inoue K-I, Adachi K, et al. The Hippo signaling pathway components Lats and Yap pattern Tead4 activity to distinguish mouse trophectoderm from inner cell mass. Dev Cell. 2009:16(3):398-410.

\section{SUPPORTING INFORMATION}

Additional supporting information may be found online in the Supporting Information section.

How to cite this article: Zhang T, Guo S, Zhou H, et al. Endometrial extracellular matrix rigidity and IFN $\tau$ ensure the establishment of early pregnancy through activation of YAP. Cell Prolif. 2021;54:e12976. https://doi.org/10.1111/cpr.12976 